\title{
Immunostimulatory bacterial antigen-armed oncolytic measles virotherapy significantly increases the potency of anti-PD1 checkpoint therapy
}

\author{
Eleni Panagioti, ${ }^{1,2,3}$ Cheyne Kurokawa, ${ }^{1,4}$ Kimberly Viker, ${ }^{1,2}$ Arun Ammayappan, ${ }^{1,2}$ S. Keith Anderson, ${ }^{5}$ Sotiris Sotiriou, ${ }^{6}$ \\ Kyriakos Chatzopoulos, ${ }^{6}$ Katayoun Ayasoufi, ${ }^{7}$ Aaron J. Johnson, ${ }^{7}$ Ianko D. Iankov, ${ }^{1,2}$ and Evanthia Galanis ${ }^{1,2}$
}

'Department of Molecular Medicine and ${ }^{2}$ Department of Oncology, Mayo Clinic, Rochester, Minnesota, USA. ${ }^{3}$ Harvey W. Cushing Neuro-oncology Laboratories (HCNL), Department of Neurosurgery, Harvard Medical School and Brigham and Women's Hospital, Boston, Massachusetts, USA. ${ }^{2}$ Department of Internal Medicine, Yale University School of Medicine, New Haven, Connecticut, USA. ${ }^{5}$ Department of Quantitative Health Sciences, ${ }^{6}$ Department of Laboratory Medicine and Pathology, and ㄱDepartment of Immunology, Mayo Clinic, Rochester, Minnesota, USA.

\begin{abstract}
Clinical immunotherapy approaches are lacking efficacy in the treatment of glioblastoma (GBM). In this study, we sought to reverse local and systemic CBM-induced immunosuppression using the Helicobacter pylori neutrophil-activating protein (NAP), a potent TLR2 agonist, as an immunostimulatory transgene expressed in an oncolytic measles virus (MV) platform, retargeted to allow viral entry through the urokinase-type plasminogen activator receptor (UPAR). While single-agent murine anti-PD1 treatment or repeat in situ immunization with MV-s-NAP-uPA provided modest survival benefit in MV-resistant syngeneic GBM models, the combination treatment led to synergy with a cure rate of $80 \%$ in mice bearing intracranial CL261 tumors and $72 \%$ in mice with CT-2A tumors. Combination NAP-immunovirotherapy induced massive influx of lymphoid cells in mouse brain, with $C D 8^{+} \mathrm{T}$ cell predominance; therapeutic efficacy was $C D 8^{+} \mathrm{T}$ cell dependent. Inhibition of the IFN response pathway using the JAK1/JAK2 inhibitor ruxolitinib decreased PD-L1 expression on myeloid-derived suppressor cells in the brain and further potentiated the therapeutic effect of MV-s-NAP-uPA and anti-PD1. Our findings support the notion that MV strains armed with bacterial immunostimulatory antigens represent an effective strategy to overcome the limited efficacy of immune checkpoint inhibitor-based therapies in CBM, creating a promising translational strategy for this lethal brain tumor.
\end{abstract}

\section{Introduction}

Glioblastoma (GBM) is the most common and lethal primary malignant brain tumor in adults, with median overall survival of 16-18 months despite treatment with surgery, radiation, and chemotherapy (1). GBM is associated with immunosuppression both systemically and in the tumor microenvironment (TME). New strategies are needed to restore antitumor immunity by modulating the GBM TME and overcome multiple immunosuppressive pathways $(2,3)$.

Translational data from early-phase clinical trials have established oncolytic viruses (OVs) as safe for brain tumor treatment and support the rationale and feasibility of virotherapy approaches to revert immunosuppression in GBM. OVs target tumor cells through unique mechanisms of action compared with currently available treatments (4). First, OVs can selectively replicate within cancer cells, resulting in direct tumor cell destruction and lysis while avoiding surrounding healthy-tissue damage. Second, several OVs have the potential to stimulate cancer cell secretion of immunogenic cell mediators by extracellular release of specific damage-associated molecular patterns (DAMPs) such as highmobility group box1 protein (HMGB1), adenosine triphosphate

Conflict of interest: The authors have declared that no conflict of interest exists. Copyright: () 2021, American Society for Clinical Investigation.

Submitted: June 23, 2020; Accepted: May 24, 2021; Published: July 1, 2021.

Reference information: J Clin Invest. 2021;131(13):e141614.

https://doi.org/10.1172/JCl141614.
(ATP), calreticulin (CALR), and heat shock protein 90 (HSP90) from dying cells. Induction of immunogenic cell death (ICD) is a powerful antitumor strategy to boost antigenicity of tumor cells and trigger an antigen-specific immune response. Third, OVs reinforce host systemic innate and adaptive immune responses through secretion of proinflammatory cytokines and increase of antigen-presenting molecules, leading to recruitment of diverse effector immune cell types in the tumor area (5).

The oncolytic properties of measles virus (MV) and advances in genetic engineering have enabled MV Edmonston (MV-Edm) vaccine strain derivatives to rapidly evolve into a promising anticancer therapy. Recombinant MVs have demonstrated encouraging durable response rates in preclinical patient-derived GBM models and remarkable safety in early-phase trials in humans (6, 7). In addition, we have recently demonstrated that activation of the IFN response pathway in patient tumors can predict permissiveness and response to oncolytic measles virotherapy $(8,9)$. However, murine cells do not express the natural MV receptors (CD46, nectin-4, and SLAM) and thus do not allow MV entry and replication in transformed cells. To circumvent receptor restriction barriers, MV strains, retargeted to enter murine cells via different receptors, are suitable platforms to allow characterization of issues such as safety, selectivity, tissue distribution, and antitumor efficacy of oncolytic MV strains in syngeneic models with intact immune systems (10). Urokinase plasminogen activator receptor (uPAR) is a key regulator of plasminogen-induced extra- 
A

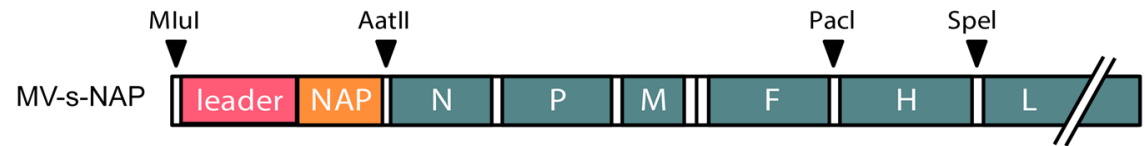

MV-GFP
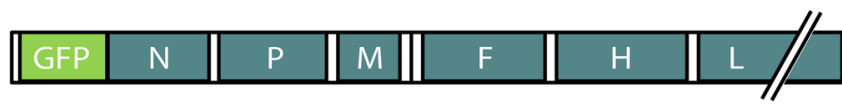

MV-s-NAP-uPA

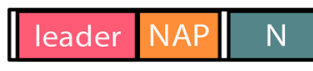

I

M

$\mathrm{H}$
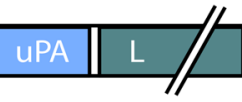

MV-GFP-uPA
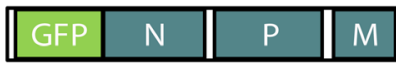

$\mathrm{F}$
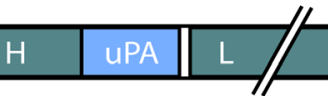

B

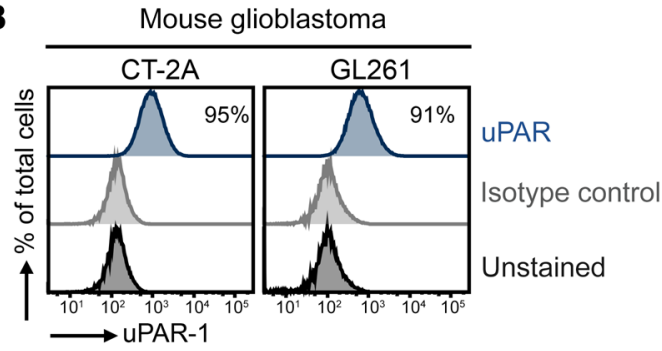

C

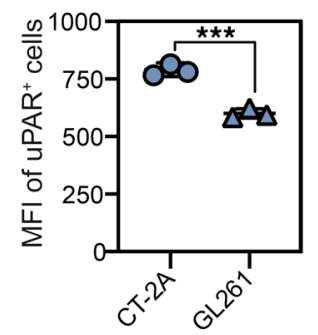

Figure 1. Schematic representation of the recombinant measles virus (MV) oncolytic vaccine platforms. (A) The Edmonston vaccine strain of the MV backbone was modified to generate the MV-s-NAP, MV-GFP, MV-s-NAPUPA, and MV-GFP-uPA recombinant strains. Where indicated, the virus was modified to encode secretory NAP protein, GFP reporter, and murine uPA ligand. The $N, P, M, F, H$, and $L$ in the virus genome diagram correspond to $M V$ proteins: nucleoprotein $(\mathrm{N})$, phosphoprotein $(P)$, matrix $(M)$ protein, fusion $(F)$ protein, hemagglutinin $(H)$, and large (L) protein. (B) Stable expression of murine $U$ PA receptor 1 (UPAR-1) was measured in CT-2A and CL261 syngeneic murine glioblastoma cells in culture after 20 cell passages. (C) Mean fluorescence intensity (MFI) of uPAR expression in CT-2A and CL261 cells. The experiment was repeated twice with similar outcomes. Values represent mean $\pm \operatorname{SD}\left(n=3\right.$ /group). ${ }^{* *} P<$ 0.001 by 2 -tailed, unpaired $t$ test. cellular proteolysis, which controls cell migration and invasion and is a biologically promising cell surface target for OV therapies (11). uPAR displays minimal expression in healthy tissues but it is naturally overexpressed in a variety of human and murine GBM cells and stroma in a similar manner, and its expression has been associated with poorer prognosis (12-14).

Preliminary evidence suggests that combined administration of OVs and bacterial components can enhance induction of antitumor responses $(15,16)$. Helicobacter pylori neutrophil-activating protein (NAP) is a major virulence factor in $H$. pylori infection (17). NAP acts as a Toll-like receptor 2 (TLR2) agonist and potent Th1-type response immunomodulator, capable of boosting immunogenicity of virus-vectored vaccines $(18,19)$. Our group has first proposed NAP as an immunostimulatory transgene for oncolytic immunotherapy (20). In proof-of-principle studies, we demonstrated that local treatment with MV strains expressing secretory NAP improved survival in patient-derived xenograft models (PDXs) of metastatic breast cancer (21).

Although large numbers of tumor-infiltrating lymphocytes (TILs) can be generated following infection with armed OVs, these $\mathrm{T}$ cells may not readily destroy tumor targets in vivo because they encounter an immunosuppressive tumor milieu. Increased activation of the Janus kinase/signal transducer and activator of transcription (JAK/STAT) signaling pathway and its upstream activators leads to upregulation of pro-proliferative, antiapoptotic, and immunosuppressive proteins, potentially driving oncogenesis $(22,23)$. JAK inhibitors are being actively tested in clinical trials as monotherapy or in combination with other agents in patients with solid tumors and hematologic malignancies. Ruxolitinib is a potent JAK1 and JAK2 inhibitor that is Food and Drug Administration (FDA) approved for the treatment of myeloproliferative diseases $(24,25)$. JAK1/JAK2 inhibition therapy with ruxolitinib represses IFN-mediated inflammatory processes elicited by viral infection or cancer $(26,27)$. Ruxolitinib has been shown to inhibit tumor growth and sensitize tumor cells to chemotherapy, immunotherapy, and OV therapy (28-30).

In this study, we sought to tackle local and systemic immune suppression of GBM with bacterial antigen-armed OV therapy and systemic immunomodulation. To this end, we engineered an MV vaccine strain to encode the secretory NAP antigen (MV-s-NAP) and retargeted hemagglutinin which allows entry in murine cells via UPAR. The antitumor activity of MV-s-NAP-uPA was evaluated in immunocompetent C57BL/6 mice harboring orthotopic CT-2A or GL261 glioblastoma and in athymic nude mice harboring orthotopic patient-derived GBM6 and GBM12 glioblastoma PDXs selected for their difference in permissiveness in MV infection. Our findings show that the combination of MV-s-NAP virotherapy with anti-programmed cell death 1 (anti-PD1) checkpoint blockade immunotherapy and the additional immunomodulatory effect of JAK1/JAK2 inhibition represents an effective strategy to heighten antitumor immune responses instigated by the virus and to overcome the limited efficacy of immune checkpoint inhibitors in GBM, therefore representing a promising therapeutic avenue for this lethal brain tumor.

\section{Results}

$M V-s-N A P-u P A$ construction and cytopathic properties. A bacterial antigen-armed MV-Edm vaccine strain fully retargeted against the murine UPAR was designed as described in Figure 1A. The uPAR-retargeted MV-GFP and MV-s-NAP strains were propagated in Vero cells and successfully rescued, leading to comparable viral titers. Next, the in vitro cytopathic potential of the uPARretargeted MV strains was thoroughly examined against the syngeneic CT-2A and GL261 glioblastoma murine cell lines. Stable 
A

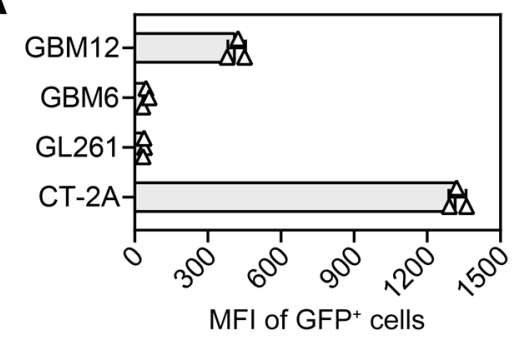

B

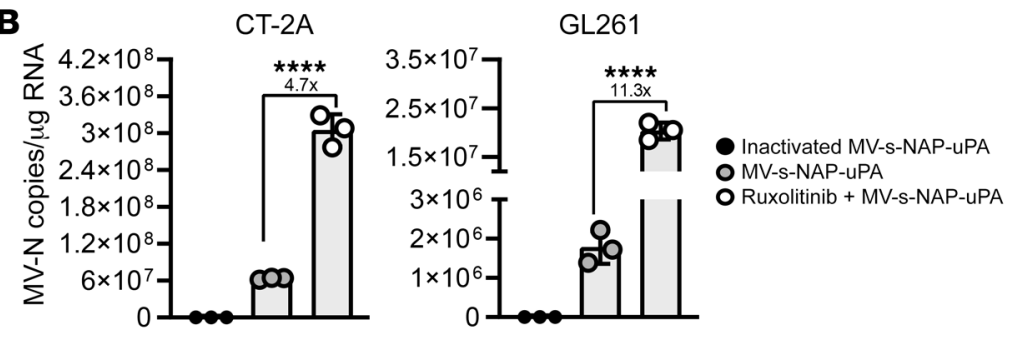

C $72 \mathrm{~h}$

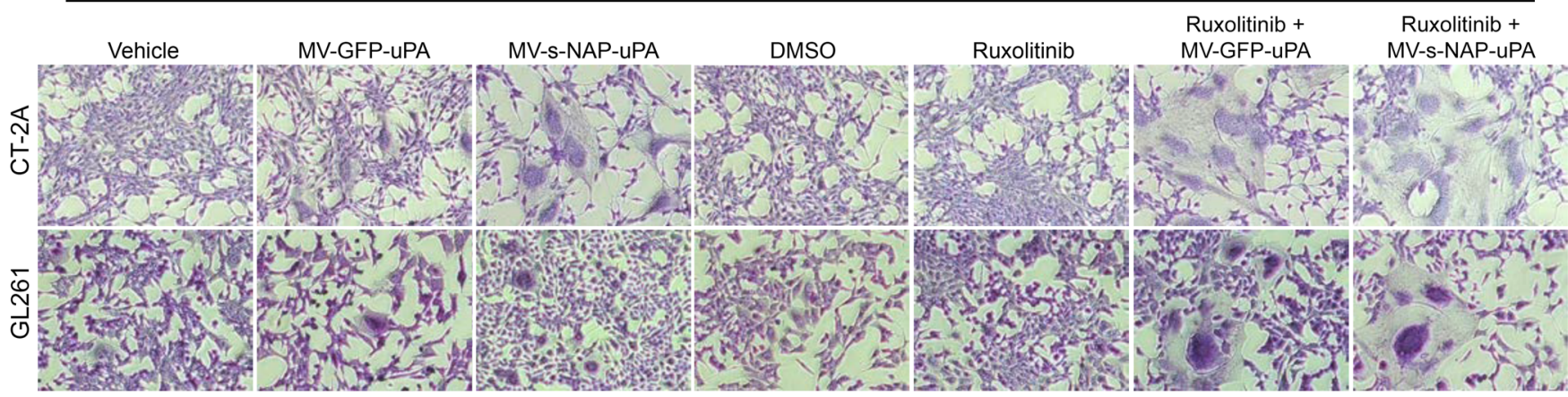

$72 \mathrm{~h}$

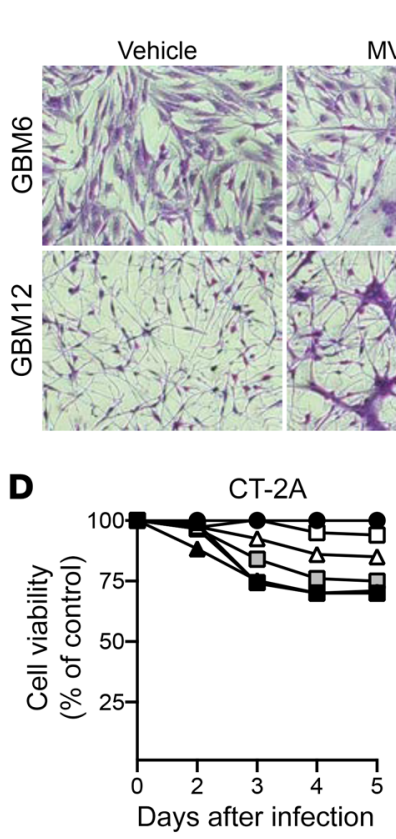

MV-GFP

MV-s-NAP

DMSO

Ruxolitinib

Ruxolitinib +

MV-GFP

Ruxolitinib +

MV-s-NAP

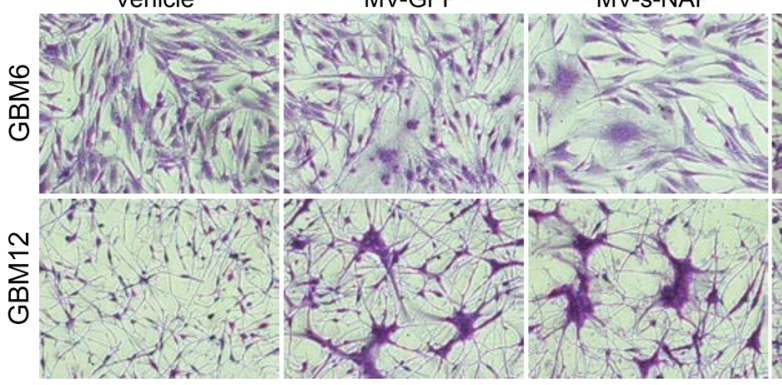

D

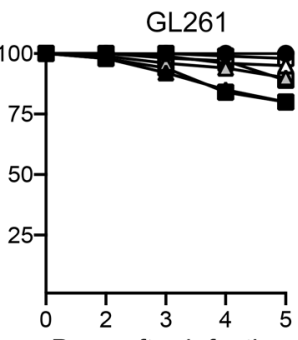

Days after infection

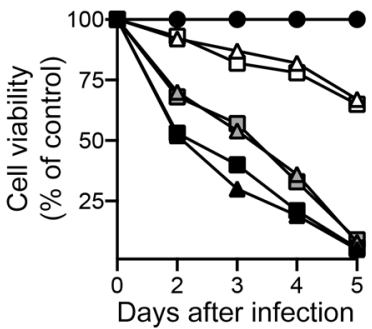

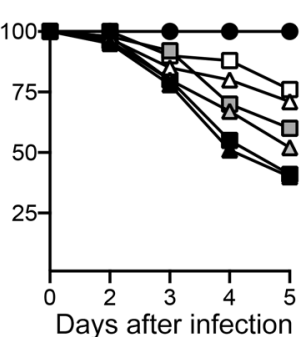

- Vehicle

$\downarrow$ MV-GFP-UPA, MOI: 2

$\triangle$ MV-GFP-UPA, MOI: 1

$\checkmark$ MV-GFP-uPA, MOI: 0.1

$\rightarrow$ MV-s-NAP-uPA, MOI: 2

- - MV-s-NAP-uPA, MOI: 1

- - MV-s-NAP-uPA, MOI: 0.1

- Vehicle

- Ruxolitinib

- MV-GFP-UPA, MOI: 2

$\triangle$ MV-GFP-UPA, MOI: 1

$\triangle$ MV-GFP-UPA, MOI: 0.1

MV-s-NAP-UPA, MOI: 2

ㅁ. MV-s-NAP-uPA, MOI: 1

$\square$ MV-s-NAP-UPA, MOI: 0.1
E
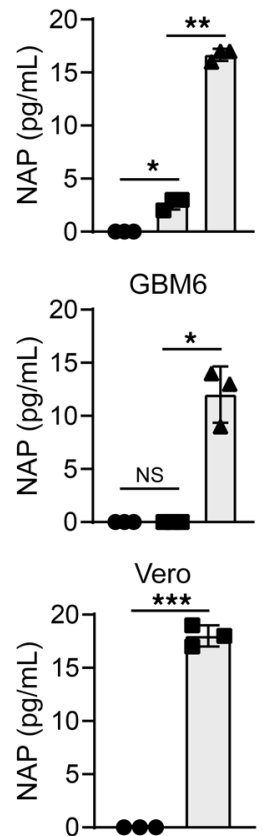

GL261
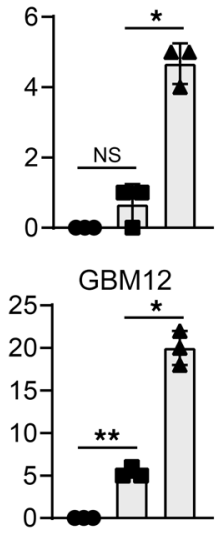

- Vehicle

- MV-s-NAP-UPA

- MV-s-NAP-UPA + Ruxolitinib

Figure 2 
Figure 2. Infection with uPAR-retargeted MV-Edm strains results in cytopathic effects against syngeneic murine and patient-derived glioblastoma lines. (A) GBM6, GBM12, CT-2A, or GL261 cells were infected in vitro with MV-GFP-uPA or MV-GFP at MOI $=2$. Expression of GFP-positive cells was measured by flow cytometry 48 hours after infection. (B) Copies of MV nucleoprotein mRNA per microgram of total RNA measured in CT-2A and GL261 cells infected at $\mathrm{MOI}=2$ with inactivated MV-s-NAP-uPA or MV-s-NAP-uPA. Where indicated, cells were pretreated with $5 \mu \mathrm{M}$ ruxolitinib and remained in the presence of the drug until completion of the experiment. Results were obtained 48 hours after infection. (C) Murine and PDX glioblastoma cells were infected with the indicated viruses at $\mathrm{MOI}=2$. Where mentioned, cells were cotreated with $5 \mu \mathrm{M}$ ruxolitinib. Following a 72 -hour incubation, virus oncolytic activity was captured by crystal violet staining. Representative light microscopy pictures of virus infection. Vehicle-treated (Opti-MEM), DMSO-treated, and ruxolitinib-treated cells were included at the assay to serve as controls. Original magnification, $\times 100$. (D) uPAR-retargeted MV infection cell viability time kinetics against CT-2A and CL261 glioblastomas measured by CellTiter-Blue assay at $\mathrm{MOI}=0.1,1$, and 2 with and without cotreatment with ruxolitinib. (E) Expression of NAP transgene $(\mathrm{ng} / \mathrm{mL})$ examined by ELISA in the cell lysates of glioma cells following 72 hours of infection with uPAR-retargeted MV-sNAP or preclinical grade MV-s-NAP at MOI $=2$ in the presence or not of $5 \mu \mathrm{M}$ ruxolitinib. MV-producing Vero cells infected with MV-s-NAP-uPA served as a positive control for NAP detection. All samples were run in triplicate and are representative of at least 2 independent experiments. Values represent mean $\pm \mathrm{SD} .{ }^{*} P<0.05 ;{ }^{* *} P<0.01 ;{ }^{* *} P<0.001 ;{ }^{* * *} P<0.0001$ by 1-way ANOVA with Tukey's multiple comparison test. NS, not significant.

constitutive overexpression of uPAR was measured in both CT-2A and GL261 cells (Figure 1, B and C). uPAR-retargeted MV-GFP virus exhibited moderate capacity to infect CT-2A and minimal capacity to infect GL261 cells as assessed by FACS analysis for GFP expression 48 hours after infection at an MOI of 2 (Figure 2A). These results reflect differences in permissiveness in MV replication between the two GBM murine models. In recent studies, we have demonstrated that permissiveness of glioma cells to MV infection is determined by baseline activation of IFN-stimulated genes (ISGs) (8). The JAK/STAT pathway plays a key role in regulating ISG transcription (31). Pretreatment of glioma cells with the JAK1/JAK2 inhibitor ruxolitinib (Jakafi) at $5 \mu \mathrm{M}$ dramatically improved virus-mediated oncolytic properties compared with control, vehicle-treated (media), and DMSO-treated cells, increasing viral replication (Figure 2B), syncytia formation (Figure $2 \mathrm{C}$ ), and oncolytic cell death in a virus dose-dependent manner (Figure 2D). The peak of viral replication occurred at 72 hours after infection for all recombinant MV strains. Although eventually both viruses reached similar infection patterns, MV-s-NAP-uPA exhibited superior lytic potential in terms of faster induction of its cytopathic effect compared with MV-GFP-uPA in all tested GBM cell lines. The NAP transgene was efficiently secreted by murine CT-2A and GL261 and human GBM6 and GBM12 cells infected with MV-s-NAP-uPA and MV-s-NAP strains, respectively (Figure $2 \mathrm{E})$. These findings encouraged concomitant administration of ruxolitinib as a strategy to overcome limitations in viral replication in MV-resistant glioma cells.

Of note, histologic examination of orthotopically implanted CT-2A tumors in immunocompetent C57BL/6 mice treated with a prime-boost intratumoral injection dose of $2 \times 10^{5} \mathrm{TCID}_{50}$ uPAR-retargeted MV-NAP revealed productive viral infection, as evidenced by the detection of tumor cell fusion and polynuclear cell formation in hematoxylin and eosin-stained (H\&E-stained) paraffin-embedded tumor sections (Supplemental Figure 1, A and $\mathrm{B}$; supplemental material available online with this article; https:// doi.org/10.1172/JCI141614DS1). Oral administration of $50 \mathrm{mg} / \mathrm{kg}$ ruxolitinib increased TIL recruitment at the tumor site but did not increase syncytial formation or intratumoral viral replication (Supplemental Figure 1, C and D).

$M V-s-N A P$ induces immunogenic cell death and proinflammatory cytokine release in syngeneic murine models of GBM. It has become increasingly clear that in addition to their direct tumoricidal effects, the ability of OVs and bacteria to induce ICD can greatly affect their potential to mount antitumor immunity (32).
Therefore, the ability of uPAR-retargeted MV strains to induce immunostimulatory cell death by extracellular release of DAMPs was examined in murine CT-2A and GL261 and in primary GBM6 and GBM12 infected cells in culture. Cell surface expression of CALR and HSP9O (both danger signals to antigen-presenting cells) and elevated extracellular release of the late-stage apoptotic biomarker HMGB1 (activation signal for immune cells) were measured in the supernatant collected from CT-2A and GL261 cells infected with uPAR-retargeted MV strains. Notably, NAParmed MV exhibited greater capacity for DAMP release compared with GFP-expressing control viruses. Pretreatment with 5 $\mu \mathrm{M}$ ruxolitinib led to rapid CALR and HSP90 cell surface export and subsequently more active release of HMGB1, suggesting that production of DAMPs is strongly associated with the capacity of MVs to replicate in immunocompetent GBM models (Figure 3, A-C). Collectively, uPAR-retargeted MV-s-NAP is a potent inducer of ICD linked to production of DAMPs when applied alone and ruxolitinib as a combinatorial strategy can enhance virus lytic growth and increase mediators of inflammation and immune stimulation in vitro.

Other proinflammatory and immunoregulatory mediators induced by UPAR-retargeted MV strains in vitro were also explored. MV-s-NAP-uPA infection increased secretion of the proinflammatory IL- $1 \beta$ and the immunoregulatory IL- 6 and IL-10 cytokines by the CT-2A and GL261 glioma cells. Elevated levels of the proinflammatory CXCL1, RANTES, and CXCL10 chemokines were also measured (Figure 3D). Viral infection resulted in dose-dependent increase in type I IFN (IFN- $\beta$ ) secretion; however, IFN- $\alpha$ secretion was not detected in the cell supernatant of infected CT-2A and GL261 cells at various time points (Supplemental Figure 2A). In comparison, MV-s-NAP-uPA infection triggered lower production of IFN- $\beta$ in CT-2A and GL261 glioma and in J774.1 macrophage murine cells that was detectable at a later infection time point, when compared with control GFP virus. Conversely, MV-s-NAP-uPA evoked stronger cytotoxicity than MV-GFP-uPA during the first 48 hours of infection against all murine glioma cell lines tested.

Although many TLR agonists drive IFN type I production, TLR2 agonists do not represent a major driver (33). Because the recombinant MV-uPA virus encodes NAP, a known TLR2 agonist, we speculated that TLR2 signaling directly or through release of proinflammatory cytokines impaired type I IFN induction and thereby frontline defense against the virus. Interestingly, MV-GFPuPA downregulated IFN- $\alpha$ and $-\beta$ receptor subunit 1 (IFNAR-1) and 
A

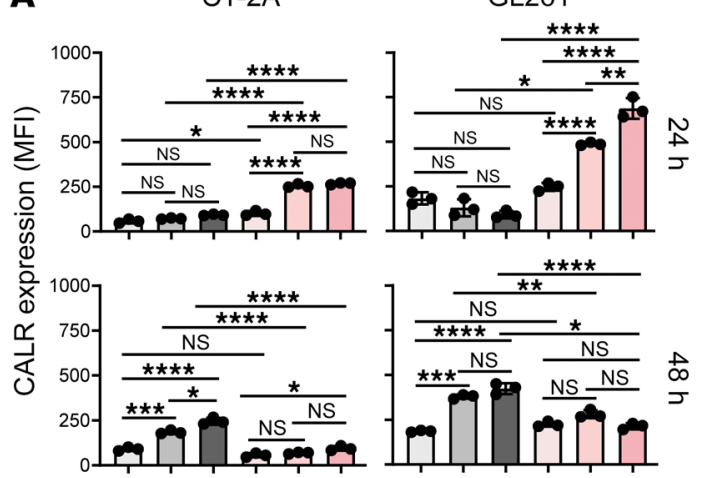

C
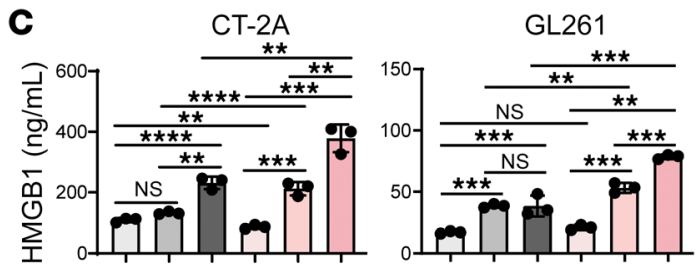

B

CT-2A

GL261

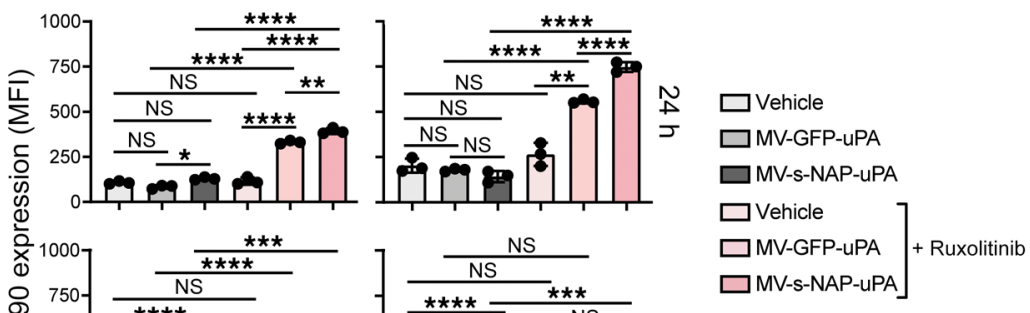

D CT-2A
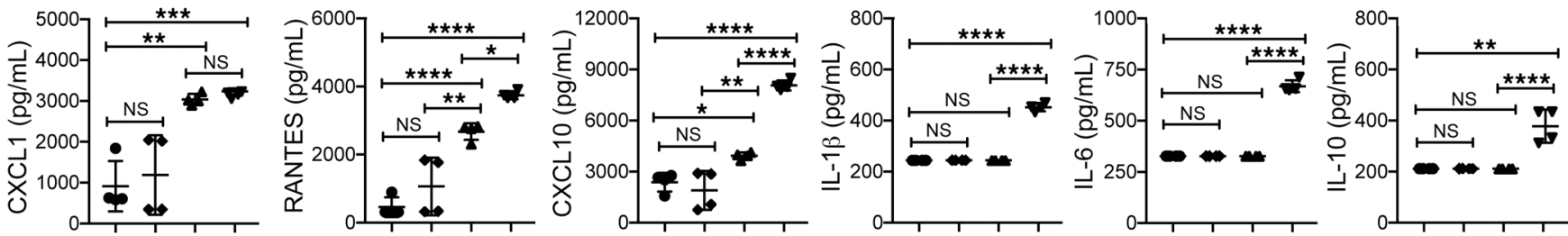

GL261
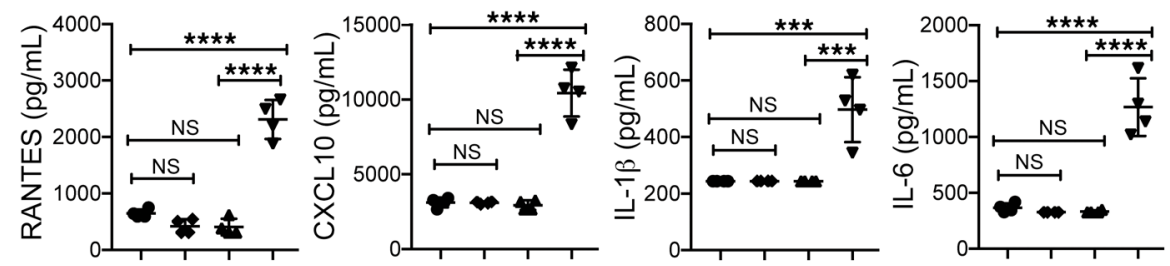

- Vehicle

- Inactivated MV-s-NAP-uPA

$\triangle \mathrm{MV}$-GFP-UPA

$\nabla$ MV-s-NAP-uPA

Figure 3. Infection with MV-s-NAP induces immunogenic cell death and release of proinflammatory mediators. CT-2A and CL261 cells were infected with the indicated viruses at $\mathrm{MOI}=5$. Cells were cotreated with $5 \mu \mathrm{M}$ ruxolitinib, where indicated. Extracellular release of (A) calreticulin (CALR) and (B) HSP9O was examined by flow cytometry 24 and 48 hours after infection. (C) CT-2A, GL261, GBM6, and GBM12 cells were infected with the indicated viruses at $\mathrm{MOI}=5$ and 72 hours later, HMGB1 release was quantified using the particle-free cell culture supernatant. (D) Cytokine and chemokine secretion (pg/ $\mathrm{mL}$ ) was measured in fresh cell culture supernatants 48 hours after infection at $\mathrm{MOI}=2$. All experiments were repeated 2 or more times with similar outcomes. Values represented as the mean $\pm \mathrm{SD}$. ${ }^{*} P<0.05$; ${ }^{* *} P<0.01 ;{ }^{* *} P<0.001$; ${ }^{* * *} P<0.0001$ by 2-way ANOVA with Tukey's multiple comparison test. NS, not significant.

upregulated TLR2 surface expression in glioma cells and J774.1 innate immune cells during the first 48 hours of infection (Supplemental Figure 2, B and C). In contrast, glioma cells infected with MV-s-NAP-uPA displayed IFNAR-1 and TLR2 surface expression levels that were similar to noninfected cells, suggesting a modified viral entry mechanism that inhibits innate immune sensing, resulting in a prolonged time for viral dissemination.

Infection with MV-s-NAP led to PD-L1 upregulation and an increase in MHC class I molecules in glioma cells. Downregulation or loss of MHC class I antigen expression by cancer cells represents an important immune evasion strategy, and is associated with reduced ability of $\mathrm{CD}^{+} \mathrm{T}$ cells to recognize tumor peptides presented by MHC class I molecules and a dismal prognosis (34). We therefore compared the surface expression of PD1 ligand (PD-L1) and MHC class I and II in cultured CT-2A and GL261 glioma cells before and after infection with uPAR-retargeted MV strains. Interestingly, PD-L1 surface expression was upregulated in GBM6 and GBM12 PDXs after infection with MV-s-NAP and MV-GFP strains (Figure 4A). Similarly, PD-L1 and MHC class I were uniformly upregulated in CT-2A and GL261 glioma cells at early postinfection time points (Figure 4, B and C). Given the well-described role of the PD1/PD-L1 immunoregulatory axis in T cell exhaustion, upregulation of PD-L1 by MV-induced tumor inflammation suggests that systemic therapy with anti-PD1 checkpoint blockade antibodies could have therapeutic utility in combination with MV therapy in GBM. In agreement with published reports, MHC class 
A PDX glioblastoma
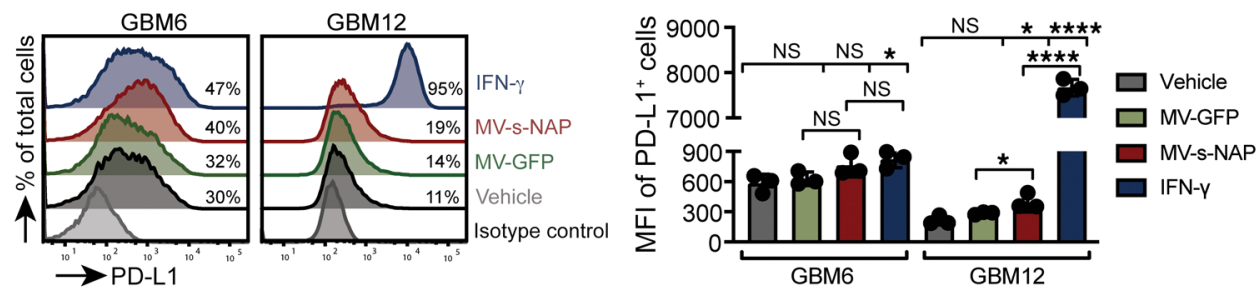

B
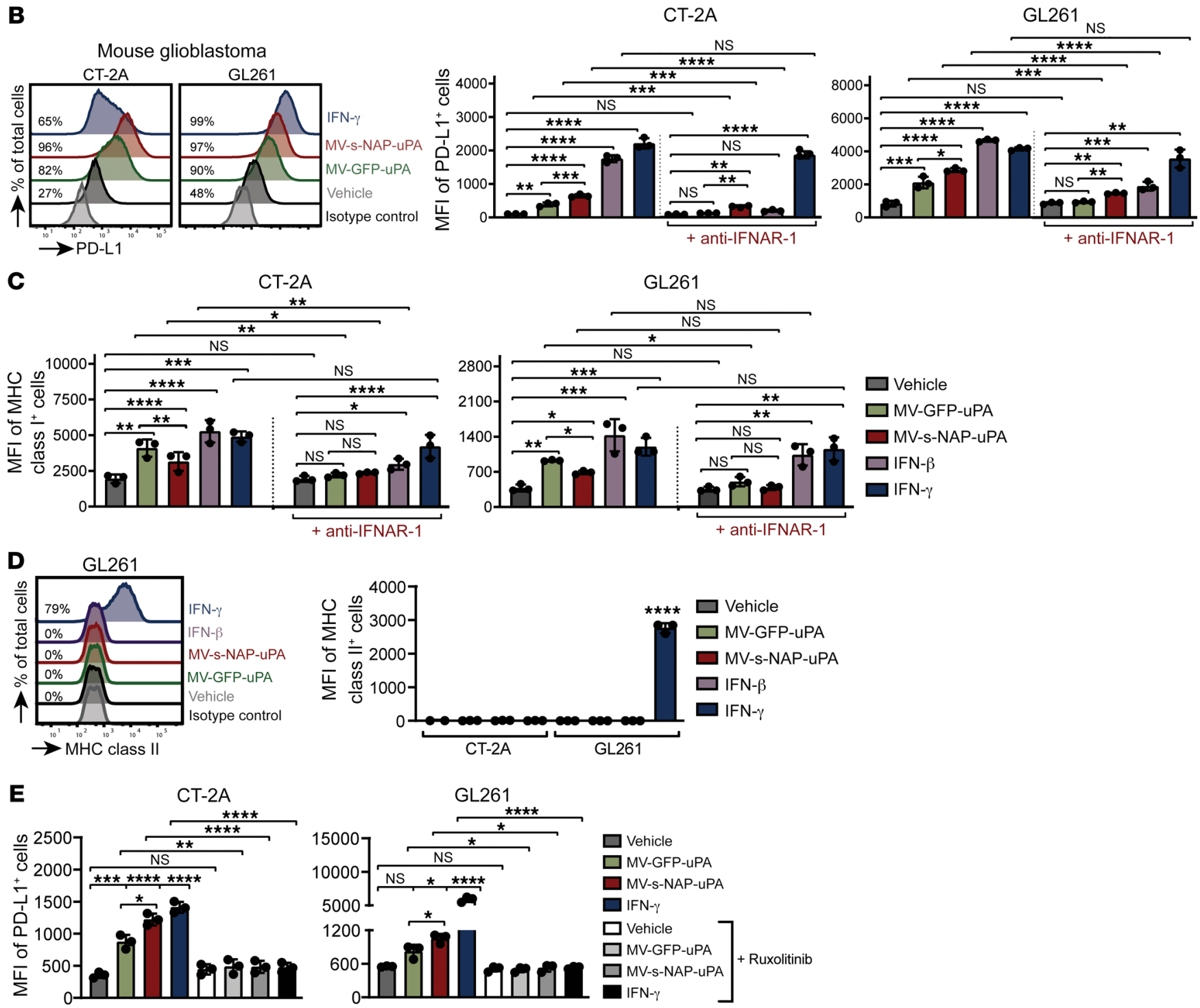

Figure 4. Infection with uPAR-retargeted MV upregulates PD-L1 and MHC class I expression in glioma cells. CT-2A, GL261, GBM6, and GBM12 cells were infected with the indicated MV strains at MOI = 2. (A) Representative histograms (left) and MFI (right) of PD-L1 surface expression assessed 48 hours after infection in GBM6 and CBM12 PDXs. (B) Representative histograms (left) and MFI (right) of PD-L1 cell surface expression assessed 48 hours after infection in CT-2A and GL261 murine glioma cells. (C) MFI of MHC class I cell surface expression in CT-2A and GL261 glioma cells infected with MV strains in the presence or absence of $10 \mu \mathrm{g} / \mathrm{mL}$ anti-IFNAR-1 depleting antibody. (D) MFI of MHC class II cell surface expression assessed 48 hours after infection in CT-2A and GL261 cells. (E) MFI of PD-L1 surface expression in CT-2A and CL261 glioma cells infected with MV strains in the presence or absence of $5 \mu$ M ruxolitinib. Glioma cells were stimulated overnight with $500 \mathrm{IU} / \mathrm{mL}$ exogenous IFN- $\beta$ or IFN- $\gamma$ to serve as controls. Values represented as the mean \pm SD and are representative of at least 2 independent experiments ( $n=3$ sets of cells/group). ${ }^{*} P<0.05 ;{ }^{* *} P<0.01 ;{ }^{* *} P<0.001$; ${ }^{* * *} P<0.0001$ by 2 -way ANOVA with Tukey's multiple comparison test. NS, not significant. 
A
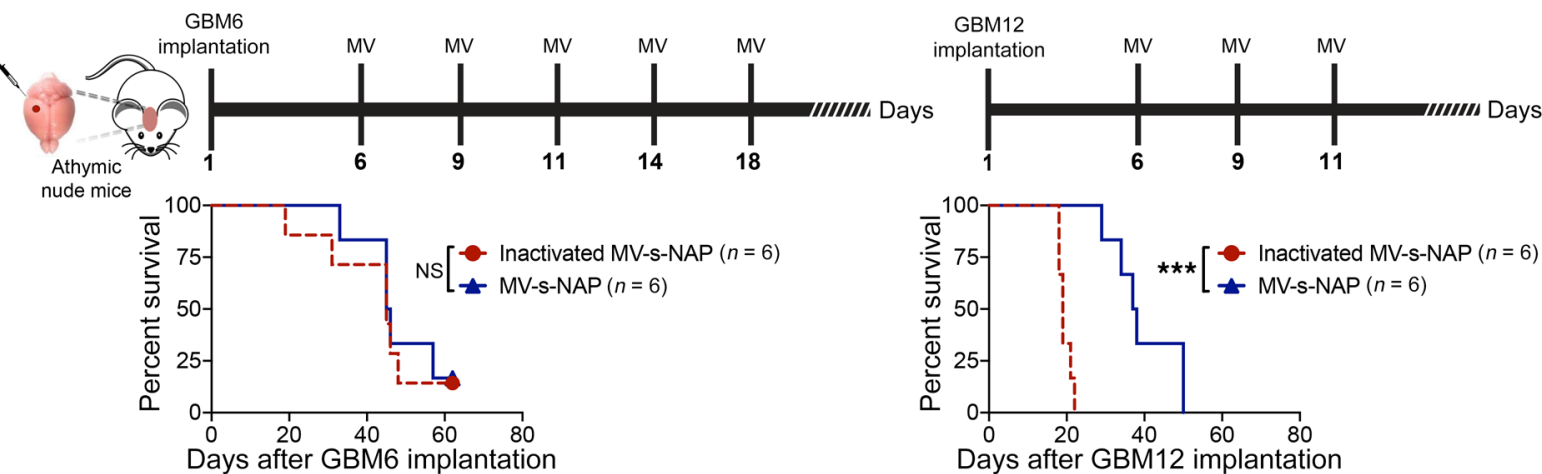

B
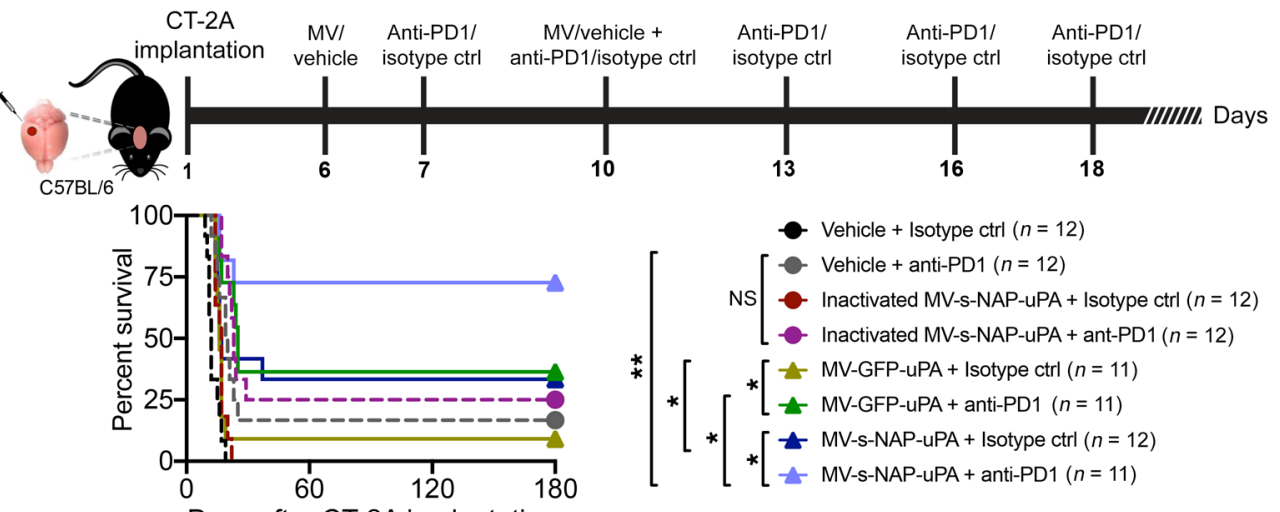

Days after CT-2A implantation
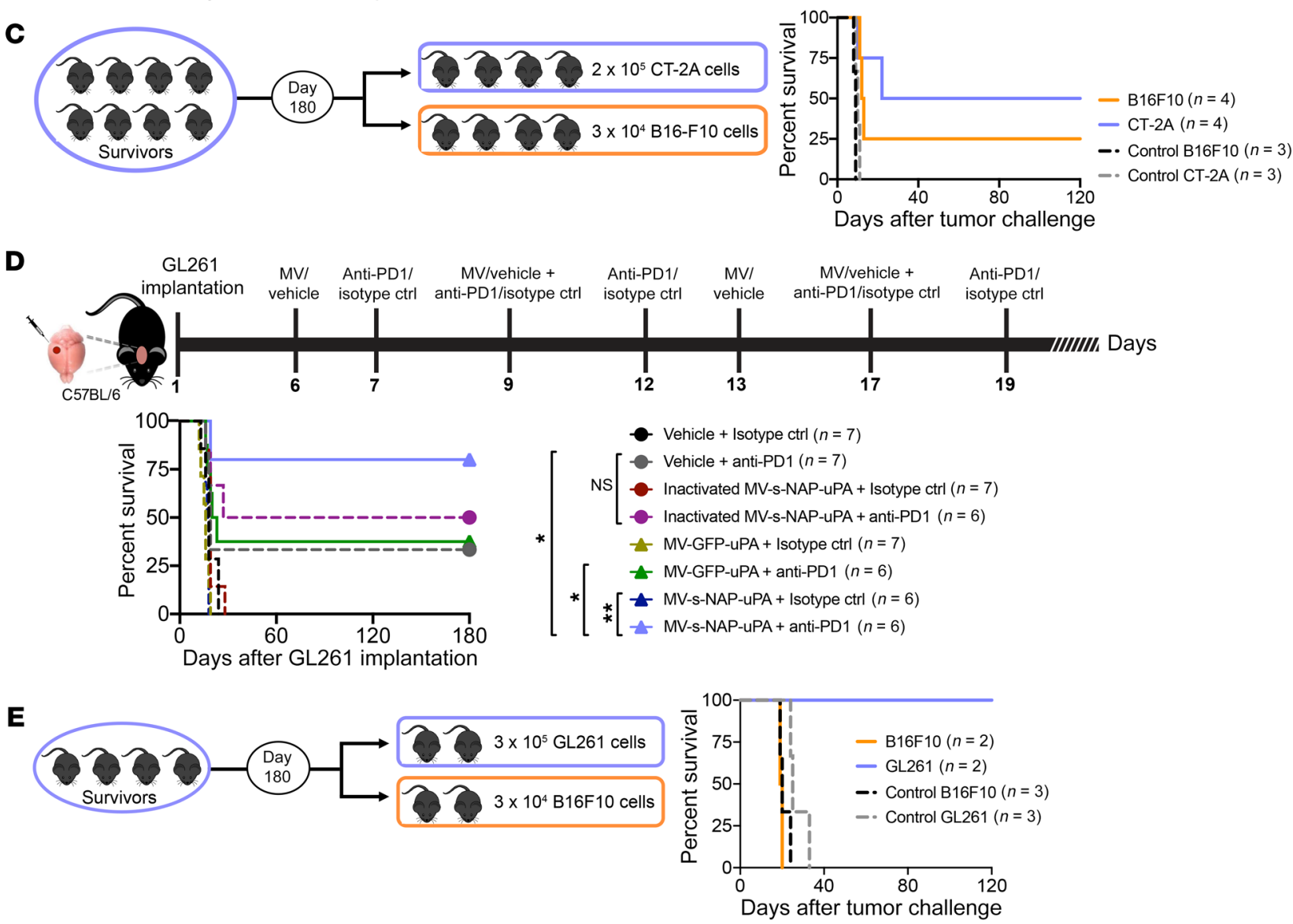

Figure 5 
Figure 5. MV-s-NAP-uPA in combination with anti-PD1 results in synergistic therapeutic efficacy against glioblastoma. (A) Survival outcomes in athymic nude mice implanted orthotopically with GBM6 or GBM12 cells and treated with intratumoral administration of MV-s-NAP-uPA or heat-inactivated MV-sNAP-uPA $\left(2 \times 10^{5} \operatorname{TCID}_{50}\right)$ once every 3 days for a total of 5 or 3 doses, respectively ( $n=6$ mice per group). (B) Survival outcomes of C57BL/ 6 mice bearing CT-2A glioma following treatment with uPAR-retargeted MV strains in combination with anti-PD1. Mouse anti-PD1 or isotype control was administered i.p. at $200 \mu \mathrm{g} /$ mouse for a total of 5 doses as per the depicted treatment schema. Inactivated MV-s-NAP-uPA virus was used as control ( $n=11-12$ mice per group). (C) Long-term-surviving CT-2A animals were rechallenged on day 180 with homologous CT-2A tumor cells or heterologous B16-F10 melanoma cells in the hemisphere contralateral to the primary injected tumor and survival was monitored ( $n=3-4$ mice per group). (D) Survival outcomes of C57BL/6 mice bearing GL261 glioma following treatment with UPAR-retargeted MV strains and anti-PD1 ( $n=6-7$ mice per group). The experiment was repeated twice with similar outcomes. (E) Long-term-surviving GL261 mice were challenged on day 180 with homologous GL261 tumor cells or heterologous B16-F10 melanoma cells in the hemisphere contralateral to the primary implanted tumor and overall survival was monitored. Kaplan-Meier survival curves and $P$ values that were determined using the log-rank Mantel-Cox test and Benjamini and Hochberg adjustment for multiple comparisons. ${ }^{*} P<0.05 ;{ }^{* *} P<0.01$;

${ }^{* * *} P<0.001$. NS, not significant. Detailed statistical differences between groups are presented in Tables 1 and 2 .

II surface expression was induced in the GL261 model following exogenous mouse IFN- $\gamma$ stimulation (Figure 4D).

We next evaluated whether IFN type I (IFN- $\alpha$ and IFN- $\beta$ ) release regulated the $\mathrm{PD}-\mathrm{L} 1$ and $\mathrm{MHC}$ class I increase in the MV-infected CT-2A and GL261 GBM cells. Anti-IFNAR-1 antibody treatment resulted in complete abrogation of MHC class I upregulation by MV-GFP-uPA and MV-s-NAP-uPA. These results suggested that type I IFN was the key mediator for the MVmediated MHC class I upregulation in vitro. Although type I IFNAR blockade completely blocked PD-L1 elevation by MV-GFPUPA infection, it only partially inhibited PD-L1 upregulation triggered by MV-s-NAP-uPA, suggesting a possible contribution of other proinflammatory cytokines in the observed effect (Figure 4, $\mathrm{C}$ and D). The JAK/STAT signaling pathway is activated by IFN type I and an array of other inflammatory cytokines and growth factors (35). Inhibition of the JAK/STAT signaling pathway with the JAK1/JAK2 inhibitor ruxolitinib abrogated the upregulation of PD-L1 mediated by uPAR-retargeted MV-NAP and IFN- $\gamma$ in both
CT-2A and GL261 glioma cells in vitro but endogenous (baseline) expression levels remained unaltered (Figure 4E).

Intratumoral $M V$-s-NAP overcomes resistance and synergizes with anti-PD1. Following the in vitro demonstration of MV infection in GBM lines, we examined the therapeutic efficacy of MV-s-NAPuPA in orthotopically implanted CT-2A and GL261 syngeneic murine models and MV-s-NAP in orthotopically implanted GBM6 and GBM12 PDXs. Taking into account the modest infection capacity of the MV strains against GL261 and GBM6 glioma cells in vitro and the robust innate immune defense OVs need to counteract in vivo, we postulated that intratumoral repetitive dosing of MV would be required to induce sufficient in situ tumor destruction and immune cell infiltration; a repetitive in situ immunization schedule consisting of multiple intratumoral/intracranial MV administrations and systemic anti-PD1 injections was deployed. To determine whether preexisting immunity to uPAR-retargeted MV-s-NAP leads to protection from secondary viral challenge, therefore limiting virus-induced inflammation, neutralizing IgG

\section{Table 1. Statistical analysis of survival outcomes in the CT-2A glioblastoma model following treatment with MV \pm anti-PD1 combination therapy}

CT-2A model

\begin{tabular}{|c|c|c|c|c|c|c|c|c|}
\hline & $\begin{array}{l}\text { Vehicle + } \\
\text { isotype ctrl }\end{array}$ & $\begin{array}{l}\text { Vehicle + } \\
\text { anti-PD1 }\end{array}$ & $\begin{array}{l}\text { Inactivated } \\
\text { MV-s-NAP-uPA } \\
\text { + isotype ctrl }\end{array}$ & $\begin{array}{l}\text { Inactivated } \\
\text { MV-s-NAP-uPA } \\
\text { + anti-PD1 }\end{array}$ & $\begin{array}{l}\text { MV-GFP-uPA + } \\
\text { isotype ctrl }\end{array}$ & $\begin{array}{c}\text { MV-s-GFP-uPA + } \\
\text { anti-PD1 }\end{array}$ & $\begin{array}{c}\text { MV-s-NAP-uPA + } \\
\text { isotype ctrl }\end{array}$ & $\begin{array}{c}\text { MV-s-NAP-uPA + } \\
\text { anti-PD1 }\end{array}$ \\
\hline $\begin{array}{l}\text { Vehicle + } \\
\text { isotype ctrl }\end{array}$ & & 0.0145 & 0.0291 & $<0.0007$ & 0.0632 & $<0.0007$ & 0.0091 & $<0.0007$ \\
\hline Vehicle + anti-PD1 & 0.0145 & & 0.0808 & 0.3050 & 0.2020 & 0.2020 & 0.6610 & 0.0196 \\
\hline $\begin{array}{l}\text { Inactivated } \\
\text { MV-s-NAP-uPA } \\
+ \text { isotype ctrl }\end{array}$ & 0.0291 & 0.0808 & & 0.0007 & 0.9795 & 0.0037 & 0.1687 & 0.0011 \\
\hline $\begin{array}{l}\text { MV-CFP-uPA + } \\
\text { isotype ctrl }\end{array}$ & 0.0632 & 0.2020 & 0.9795 & 0.0145 & & 0.0353 & 0.3076 & 0.0044 \\
\hline $\begin{array}{l}\text { MV-s-GFP-uPA } \\
+ \text { anti-PD1 }\end{array}$ & $<0.0007$ & 0.2020 & 0.0037 & 0.6450 & 0.0353 & & 0.6450 & 0.0423 \\
\hline
\end{tabular}

+ anti-PD1

The Benjamini and Hochberg false discovery rate (FDR) method was used to adjust for multiple comparisons. Statistical significance was set at FDR and $P$ values $<0.05$. 


\section{Table 2. Statistical analysis of survival outcomes in the GL261 glioblastoma model following treatment with MV \pm anti-PD1 combination therapy}

GL261 model

\begin{tabular}{|c|c|c|c|c|c|c|c|c|}
\hline & $\begin{array}{c}\text { Vehicle + } \\
\text { isotype ctrl }\end{array}$ & $\begin{array}{l}\text { Vehicle + } \\
\text { anti-PD1 }\end{array}$ & $\begin{array}{l}\text { Inactivated } \\
\text { MV-s-NAP-uPA } \\
\text { + isotype ctrl }\end{array}$ & $\begin{array}{l}\text { Inactivated } \\
\text { MV-s-NAP-uPA } \\
\text { + anti-PD1 }\end{array}$ & $\begin{array}{c}\text { MV-CFP-uPA + } \\
\text { isotype ctrl }\end{array}$ & $\begin{array}{c}\text { MV-s-GFP-uPA + } \\
\text { anti-PD1 }\end{array}$ & $\begin{array}{c}\text { MV-s-NAP-uPA + } \\
\text { isotype ctrl }\end{array}$ & $\begin{array}{c}\text { MV-s-NAP-uPA + } \\
\text { anti-PD1 }\end{array}$ \\
\hline $\begin{array}{l}\text { Vehicle + isotype } \\
\text { ctrl }\end{array}$ & & 0.2952 & 0.4645 & 0.0644 & 0.1561 & 0.2359 & 0.3656 & 0.0350 \\
\hline Vehicle + anti-PD1 & 0.2952 & & 0.0627 & 0.5316 & 0.0659 & 0.7445 & 0.0659 & 0.0403 \\
\hline $\begin{array}{l}\text { Inactivated } \\
\text { MV-s-NAP-uPA } \\
+ \text { isotype ctrl }\end{array}$ & 0.4645 & 0.0627 & & 0.1141 & 0.0644 & 0.2031 & 0.0627 & 0.0350 \\
\hline $\begin{array}{l}\text { MV-GFP-uPA } \\
+ \text { isotype ctrl }\end{array}$ & 0.1561 & 0.0659 & 0.0644 & 0.0350 & & 0.0326 & 0.5307 & 0.0322 \\
\hline $\begin{array}{l}\text { MV-s-GFP-uPA } \\
\text { + anti-PD1 }\end{array}$ & 0.2359 & 0.7445 & 0.2031 & 0.6332 & 0.0326 & & 0.0560 & 0.0414 \\
\hline
\end{tabular}

+ anti-PD1

The Benjamini and Hochberg false discovery rate (FDR) method was used to adjust for multiple comparisons. Statistical significance was set at FDR and $P$ values $<0.05$.

antibodies against MV were measured in whole blood of mice bearing GL261 tumors after the fourth intratumoral viral infectious dose. Generation of anti-MV IgG with intermediate neutralization capacity, effective in protecting against symptomatic MV disease but unable to confer sterile immunity against subsequent viral challenge, encouraged repeat intratumoral administration of NAP-armed MV in the brain (Supplemental Figure 3, A and B).

In the orthotopic athymic nude mouse GBM12 PDX model, in situ immunization with MV-s-NAP therapy resulted in a 37-day prolongation in median survival (100\% increase) compared with heat-inactivated MV-s-NAP $(P=0.0005)$. In comparison, MV-sNAP treatment of MV-resistant GBM6 PDX mice exhibited no prolongation in median survival compared to the heat-inactivated MV-NAP group $(P=0.6559)$ (Figure $5 \mathrm{~A})$. In syngeneic mouse model experiments, immunocompetent C57BL/6 mice, harboring orthotopic CT-2A or GL261 gliomas, were randomized and treated as described in the Methods section according to the treatment schedule outlined in Figure 5, B and D. Specifically, 2 doses of uPAR-retargeted MV strains were administered to the MV-permissive CT-2A model and 4 repeat doses to the MV-resistant GL261 model. In both glioma models, virus was administered every 4 days to reach replication peak kinetics and to provide mice with adequate postoperative physical functional recovery time. AntiPD1 checkpoint blockade was administered systemically 2 to 3 days apart for a total of 5 doses. Treatment with uPAR-retargeted MV-s-NAP and anti-PD1 immunovirotherapy regimens was well tolerated in C57BL/6 mice without acute systemic adverse effects or neurological toxicity being observed. Animals that received MV-s-NAP-uPA in combination with anti-PD1 showed a significant increase in survival compared with the rest of the treatment groups (Figure 5, B and D, and Tables 1 and 2). Notably, $72 \%$ and $80 \%$ of the mice were deemed long-term survivors, remaining alive for at least 180 days after CT-2A implantation $(P \leq 0.0007)$ and GL261 $(P=0.0350)$, respectively, supporting a synergistic effect of the combination in the context of modest efficacy with virus or anti-PD1 alone. Single anti-PD1 immunotherapy cured $16 \%$ and $30 \%$ of mice harboring CT-2A and GL261 tumors, respectively, highlighting the immunosuppressive role of the PD1/PD-L1 axis in GBM. Additionally, substantial benefit in survival was observed with single MV-s-NAP-uPA virotherapy, eradicating $33 \%$ of CT-2A gliomas, but no treatment effect was noted in the GL261 model, suggesting that sensitivity to virusmediated inflammation in vitro can predict sensitivity to singleagent MV virotherapy in vivo, and further highlighting the importance of direct oncolysis and virus-mediated inflammatory responses in the observed antitumor efficacy.

Surface staining for a panel of inflammatory response markers in the peripheral blood of GL261 survivor mice on day 24 after the last intracranial virotherapy delivery showed increased levels of $\mathrm{PD} 1^{+} \mathrm{CD} 4^{+}$and $\mathrm{PD} 1^{+} \mathrm{CD} 8^{+} \mathrm{T}$ cells compared with healthy nontumor-bearing mice that had received intracranial injections of saline (Supplemental Figure 4). Although PD1 expression may be considered a marker of terminally differentiated, exhausted $\mathrm{T}$ cells, a number of recent studies in patients with malignant glioma have shown that PD1 expression in peripheral blood $\mathrm{T}$ cells may in fact be a marker of chronic activation and clonal expansion of tumor antigen-specific memory $\mathrm{T}$ cells needed to maintain tumor control $(36,37)$.

To determine the durability of the observed protection, longterm CT-2A (day 180) and GL261 (day 180) tumor-surviving ani- 
A

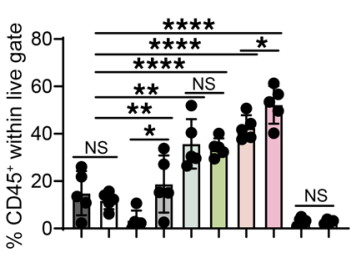

KLGR $1^{+} \mathrm{CD} 8^{+} \mathrm{T}$ cells

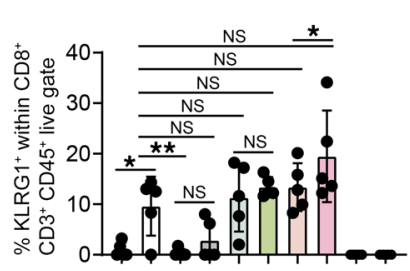

$\mathrm{PD}^{+} \mathrm{CD}^{+} \mathrm{T}$ cells
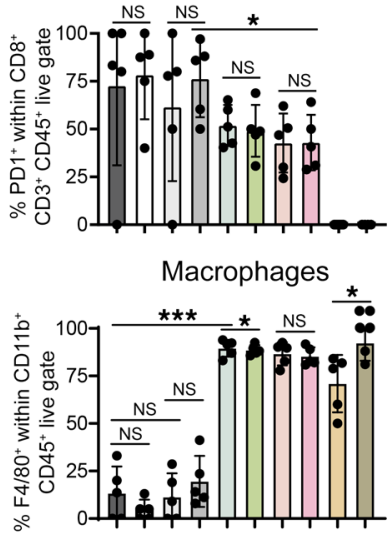

B
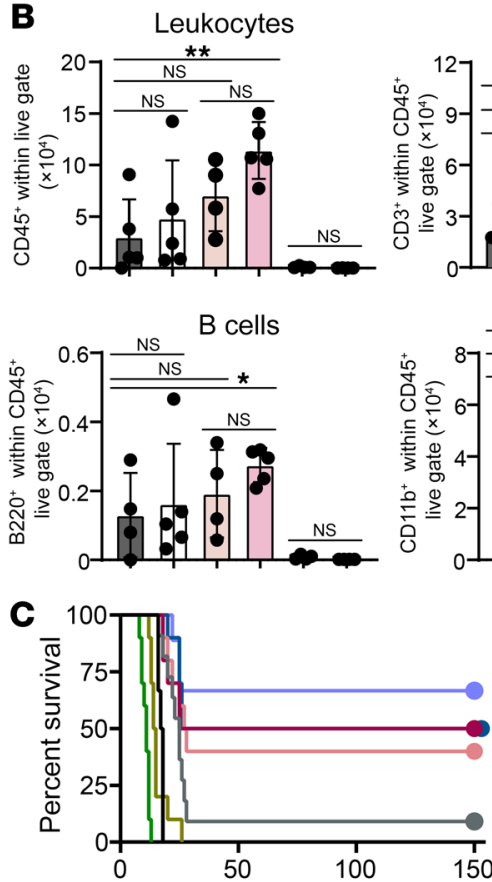

Days after CT-2A implantation

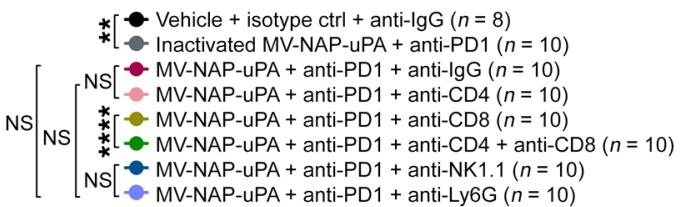

* - Vehicle + isotype ctrl + anti-lgG $(n=8)$

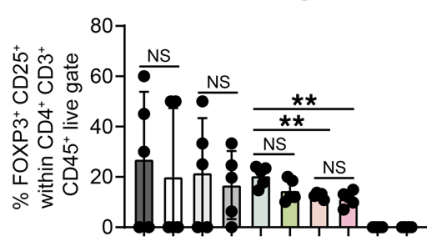

B cells

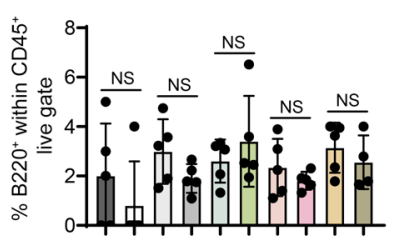

Granzyme B CD8 ${ }^{+} \mathrm{T}$ cells

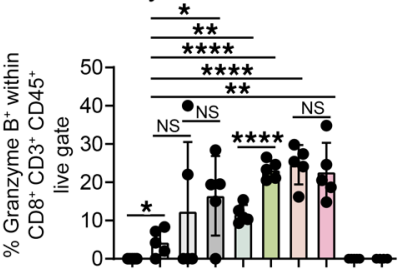

CD4 ${ }^{+}$Tregs
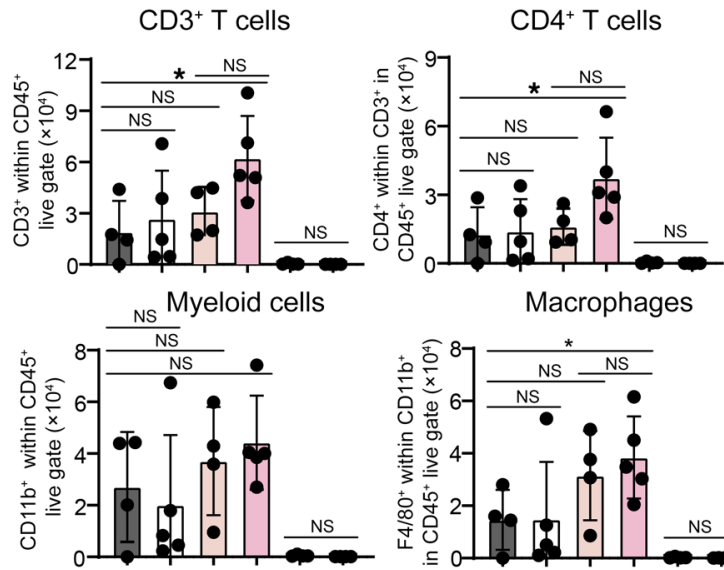

Macrophages
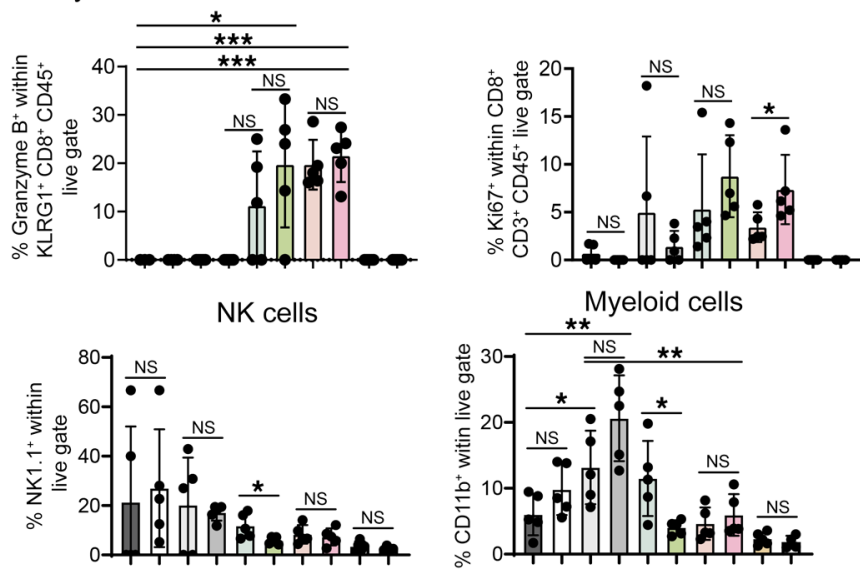

$$
\begin{aligned}
& \square \text { Vehicle + isotype ctrl }(n=5) \\
& \square \text { Vehicle + anti-PD1 }(n=5) \\
& \square \text { Inactivated + MV-s-NAP-uPA + isotype ctrl }(n=5) \\
& \square \text { Inactivated + MV-s-NAP-uPA + anti-PD1 }(n=5) \\
& \square \text { MV-GFP-uPA + isotype ctrl }(n=5) \\
& \square \text { MV-GFP-uPA + anti-PD1 }(n=5) \\
& \square \text { MV-s-NAP-uPA + isotype ctrl }(n=5) \\
& \square \text { MV-s-NAP-uPA + anti-PD1 }(n=5) \\
& \square \text { Naive + vehicle intracranially }(n=5) \\
& \square \text { Naive }(n=4)
\end{aligned}
$$

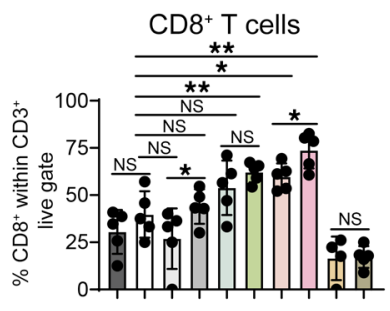

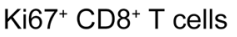


Figure 6. Intratumoral MV-s-NAP-uPA and systemic anti-PD1 immunovirotherapy results in potent cellular immune response against glioblastoma. Mice bearing (A) CT-2A and (B) GL261 orthotopic tumors were treated according to the schema in Figure 5, B and D, respectively. At the time point that at least 2 mice from the vehicle plus isotype control group exhibited clinical symptoms of CBM disease progression, all groups of mice were sacrificed for analysis of immune cell responses in the brain. Mice were perfused and brains were processed for immunophenotyping. Graph values represent mean \pm SD ( $n=4-5$ mice per group). (C) Mice bearing CT-2A brain tumors were treated with MV-s-NAP-uPA and anti-PD1 antibody as described in Figure 5B in the presence of anti-CD4, -CD8, -CD4 plus -CD8, -NK1.1, and -Ly6G cell-depleting antibodies. Kaplan-Meier survival curves and Benjamini and Hochberg adjustment for multiple comparisons were used to calculate median survival times ( $n=8-10$ mice per group). (D) Images of spleen and thymus sizes harvested from individual mice after completion of the MV-s-NAP-uPA and/or anti-PD1 treatment of the GL261 glioblastoma model. Untreated naive mice and naive mice injected with saline (vehicle) intracranially were included in the experiment as controls. ${ }^{*} P<0.05 ;{ }^{* *} P<0.01 ;{ }^{* *} P<0.001$; ${ }^{* * * *} P<0.0001$ by 2 -way ANOVA followed by Tukey's multiple comparison test. NS, not significant.

mals were challenged with autologous GBM cells or with heterologous B16-F10 melanoma cells contralaterally to the primary tumor injection site and followed for survival (Figure 5, C and E). GL261 long-term survivors were protected against rechallenge with the same tumor model but not against B16-F10 tumor challenge, suggesting that MV-s-NAP-uPA and anti-PD1 treatments were efficacious in creating a durable, long-term tumor-specific immunity. In parallel, $50 \%$ of the CT-2A long-term survivors were protected after secondary challenge with CT-2A cells and $25 \%$ after challenge with the melanoma B16-F10 cells, suggesting that the nonspecific inflammatory response component could also have played a role in protection. These data in 2 different syngeneic orthotopic models demonstrate that combination immunovirotherapy with MV-s-NAP and anti-PD1 can eradicate orthotopic GBM in the majority of the animals and establish durable tumor-specific immunity.

The therapeutic effects of intratumoral $M V-s-N A P-u P A$ rely on a functional immune system. To understand the effect of combination treatment in the TME, we performed comparative immune profiling of the CT-2A and GL261 TMEs and assessed the differences in immune infiltrates between the wild-type and treated animals using flow cytometry and histological examination. In parallel experiments, C57BL/6 mice bearing orthotopic CT-2A and GL261 tumors were treated according to the timelines summarized in Figure 5, B and D. Upon termination of the experiment, all animals were euthanized and individual brains were collected for multiple comparison analysis when at least 2 animals from the vehicle plus isotype control group reached euthanasia criteria that define progression of GBM disease (this was performed on day 9 after treatment initiation for the CT-2A model and day 20 for the GL261 model).

Mice receiving MV-s-NAP plus anti-PD1 had a higher influx of leukocytes (CD45 $5^{+}$cells), macrophages $\left(\mathrm{F} 4 / 80^{+} \mathrm{CD} 11 \mathrm{~b}^{+}\right)$, and $\mathrm{CD}^{+} \mathrm{T}$ cells into the brain compared with all other therapy groups (Figure 6A and Supplemental Figure 5). Further characterization of the $\mathrm{T}$ cell subpopulations revealed that mice treated with MV-s-NAP-uPA plus anti-PD1 had a higher percentage of $\mathrm{CD}^{+}$and $\mathrm{CD}^{+} \mathrm{T}$ cells, and increased expression of the effector immune markers KLRG1, GzmB, and Ki67 by $\mathrm{CD}^{+} \mathrm{T}$ cells. A trend toward decreased $\mathrm{PD} 1^{+} \mathrm{CD} 8^{+} \mathrm{T}$ cells and Tregs $\left(\mathrm{CD} 25^{+}\right.$ $\left.\mathrm{FOXP}^{+}{ }^{+} \mathrm{CD} 4^{+}\right)$as compared with saline treatment, treatment with heat-inactivated virus, or with either therapy alone was observed in the CT-2A model (Figure 6A). Importantly, increased percentages of $\mathrm{KLRG} 1^{+} \mathrm{CD} 8^{+} \mathrm{T}$ cells were detected in the spleen of CT-2A tumor-treated animals, suggesting peripheral $\mathrm{T}$ cell activation (Supplemental Figure 6).
To determine which components of the cellular immunity were of utmost importance for the survival benefit observed for the MV-s-NAP-uPA/anti-PD1 combination therapy group, we repeated the efficacy experiment in the presence of cell-depleting antibodies for $\mathrm{CD}^{+}$and $\mathrm{CD} 8^{+}$cells, NK cells, and neutrophils for the CT-2A model. Adequate cell depletion ( $>97 \%$ ) of each immune cell subset was confirmed by peripheral blood flow cytometry (Supplemental Figure 7). Depletion of $\mathrm{CD} 8^{+}$or both $\mathrm{CD} 4^{+}$and $\mathrm{CD} 8^{+} \mathrm{T}$ cells resulted in complete abrogation of the antitumor therapeutic effect (Figure 6B). In contrast, elimination of $\mathrm{CD} 4^{+}$cells, NK cells, and neutrophils did not result in appreciable change in antitumor activity. Moreover, to address the role of $\mathrm{CD} 8^{+} \mathrm{T}$ cells in GL261 antitumor immunity, all GL261 long-term survivor mice (day 300) that had survived homologous tumor rechallenge (Figure 5E) were treated with $\mathrm{CD} 8^{+}$cell-depleting antibodies and subsequently rechallenged intracranially with GL261 cells. None of the GL261 long-term survivor animals survived the second challenge in the group of mice in which $\mathrm{CD} 8^{+} \mathrm{T}$ cells were depleted, demonstrating that GL261-tumor immune protection relied on $\mathrm{CD}^{+} \mathrm{T}$ cells (Supplemental Figure 8, A and B). Overall, these findings highlight the important role of T cell-mediated immune responses for systemic therapeutic efficacy of MV-s-NAP-uPA plus anti-PD1 combination therapy in GBM.

Gliomas can have a systemic immunosuppressive effect on the body's immune system, often causing major involution of the spleen and thymus leading to a dramatic drop in the number of circulating $\mathrm{T}$ cells and eventual loss of adaptive immunity in experimental animals. Severe impairment in size and functionality of the thymus and spleen in the course of GBM growth has been reported (38). We therefore examined the ability of MV-s-NAP-uPA plus antiPD1 combination immunovirotherapy to reverse GL261-mediated systemic immunosuppression. Vehicle-treated animals bearing orthotopic GL261 GBM exhibited severe cellular immunodeficiency accompanied by a dramatic decrease in splenic and thymic sizes and total cell counts (Figure 6C and Supplemental Figure 9). Combination treatment reversed GL261-induced immunosupression in all treated animals. Similarly, anti-PD1-treated animals exhibited normal splenic and thymic sizes, providing a possible explanation as to why anti-PD1 monotherapy delays GL261 disease progression in some animals. In situ immunization with MV-s-NAP-uPA monotherapy was also able to reverse systemic immunosuppression in $50 \%$ of the treated animals.

Localized MV-s-NAP-uPA infection with systemic anti-PD1 leverages abscopal therapeutic effect. We further tested whether the combination of intratumoral injection of MV-s-NAP and systemic delivery of anti-PD1 has antitumor efficacy against contralateral 

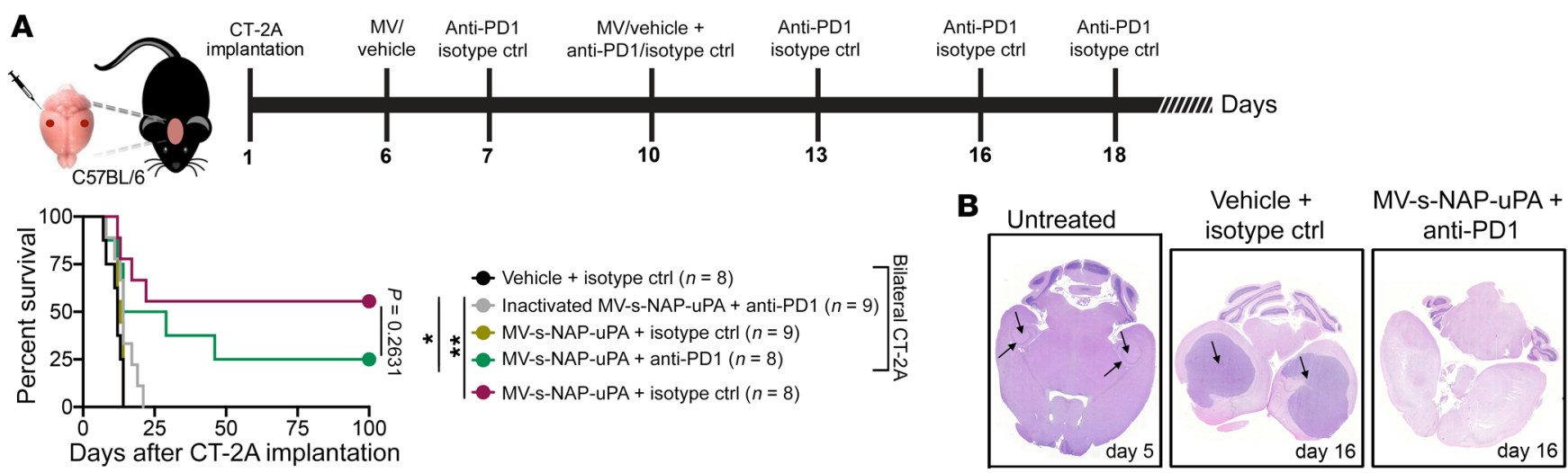

C

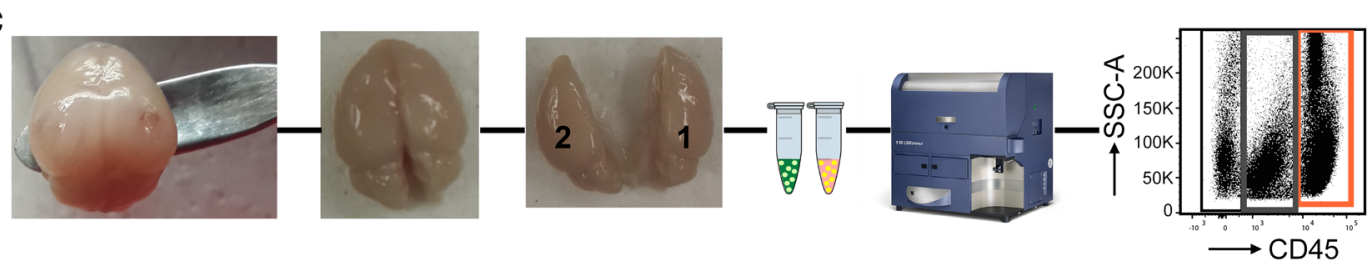

D Leukocytes
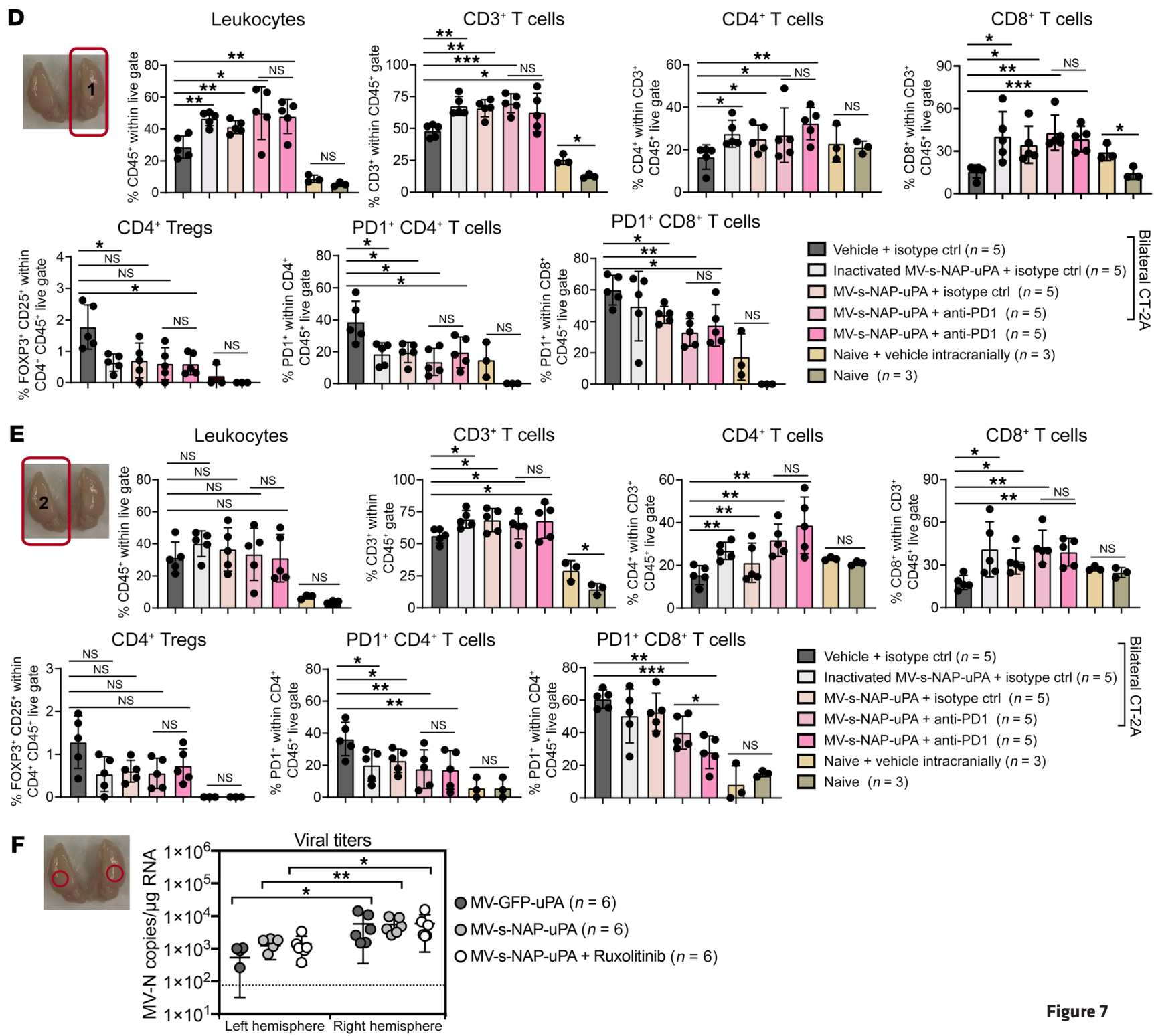

Figure 7 
Figure 7. Localized MV-s-NAP infection with systemic anti-PD1 leverages the abscopal therapeutic effect. (A) CT-2A cells were injected in the right (1 $\times$ $10^{5}$ cells) and left $\left(1 \times 10^{5}\right.$ cells $)$ hemisphere of the brain and 2 distinct bilateral CT-2A tumors were established. On day 5 after tumor engraftment, animals were randomized and tumors in the right hemisphere were treated intratumorally with MV-s-NAP-uPA as described in the treatment scheme. Tumors in the left hemisphere of the brain were left untreated. Systemic anti-PD1 blocking antibody treatment was provided as described in the Methods. Median survival of the bilateral CBM mouse model was monitored. A group of mice that was implanted with CT-2A cells only in the right side of the brain and subsequently received combination immunovirotherapy was included in the experiment to serve as treatment efficacy control ( $n=8-9$ mice per group). ${ }^{*} P<0.05$; ${ }^{* *} P<0.01$ by log-rank Mantel-Cox test and Bonferroni's correction. NS, not significant. (B) Representative H\&E-stained slides of mouse brains on day 5 and 16 after CT-2A tumor implantation. Mice received vehicle plus isotype control or MV-s-NAP-uPA plus anti-PD1 treatment. Arrows indicate areas of tumor formation. (C) In a parallel experiment, cellular immune responses in the brain of mice harboring bilateral CT-2A tumors were examined. Brains were subdivided into right (treated) and left (untreated) hemispheres and processed into single-cell suspensions. (D) Immune cell responses of treated and (E) untreated half brains ( $n=3-5$ mice per group). (F) Copies of MV nucleoprotein mRNA per microgram of total RNA measured in right and left hemispheres of CT-2A brain-tumor biopsies of mice 72 hours after treatment with MV-GFP-uPA, MV-s-NAP-uPA, or MV-s-NAP-uPA plus ruxolitinib. Each symbol represents an individual mouse. The dashed line represents the background response measured in saline-treated animals. Values represent mean \pm SD ( $n=6$ mice per group). ${ }^{*} P<0.05 ;{ }^{* *} P<0.01 ;{ }^{* *} P<0.001$ by 2 -way ANOVA followed by Tukey's multiple comparison test. NS, not significant.

CT-2A GBM. Briefly, mice were orthotopically implanted with $1 \times 10^{5} \mathrm{CT}-2 \mathrm{~A}$ cells in the right and left hemispheres of the brain, leading to the formation of 2 distinct contralateral malignant neoplasms. The right hemisphere tumor was locally treated with MV-s-NAP-uPA, while the contralateral tumor was left untreated (Figure 7A). Combination immunovirotherapy resulted in rejection of distant, noninjected primary intracranial glioma growth and long-term survival in $25 \%$ of the mice $(P=0.0134)$, suggesting an abscopal effect of the combination therapy and presence of active immunosuppressive mechanisms in the TME of the nonresponding animals. Single-agent immunotherapy with anti-PD1 failed to constrain tumor outgrowth of distant intracranial CT-2A tumors. Similarly, MV-s-NAP-uPA monotherapy provided no survival benefit in this bilateral GBM model, suggesting that a combination of virus-induced inflammation in the brain and anti-PD1 immunoregulation was critical for the abscopal treatment effect to be observed (Figure 7, A and B).

To examine the CT-2A TME in treated animals in relation to the location of the injection, right and left brain hemispheres were collected and processed individually (Figure 7C). Comprehensive flow cytometric analysis of the MV-treated right brain hemispheres revealed prominent $\mathrm{CD} 45^{+}$inflammatory infiltrate recruitment following combination immunovirotherapy. More specifically, animals demonstrated elevated numbers of $\mathrm{CD}^{+}$ and $\mathrm{CD}^{+} \mathrm{T}$ cells, and substantial decreases in Tregs, $\mathrm{PD} 1^{+} \mathrm{CD} 4^{+}$, and $\mathrm{PD} 1^{+} \mathrm{CD} 8^{+} \mathrm{T}$ cells, compared with anti-PD1 or MV-s-NAPuPA monotherapy treated (Figure 7D). Interestingly, analysis of the distant, nonvirally injected left brain hemispheres showed no increase in overall infiltrating leukocytes $\left(C D 45^{+}\right)$but a marked increase in $\mathrm{CD} 4^{+}$and $\mathrm{CD} 8^{+} \mathrm{T}$ cells and reduction in Tregs and $\mathrm{PD}^{+} \mathrm{CD}^{+}$and $\mathrm{PD} 1^{+} \mathrm{CD} 8^{+} \mathrm{T}$ cells in animals that received combination immunovirotherapy (Figure 7E). Furthermore, MV copy numbers were quantified using quantitative real-time PCR (qPCR) in both hemispheres. We were able to detect low-copy viral RNA in the noninjected hemisphere but no syncytia formation (Figure $7 F)$. Overall, these findings demonstrate the potential of a double-pronged strategy to sensitize tumors to anti-PD1 checkpoint blockade therapy, providing a strong rationale for exploration of such combination strategies in the GBM clinical setting.

Pharmacological inhibition of the JAK1/JAK2 signaling pathway with ruxolitinib improves GBM cure rates by abrogating PD-L1 expression on MDSCs. Next, we investigated whether in vivo inhibition of the JAK1/JAK2 signaling pathway with ruxolitinib would improve
MV-s-NAP-uPA and anti-PD1 combination therapy in mice bearing orthotopic CT-2A gliomas. Treatment with ruxolitinib was initiated 1 day before virus administration and was terminated 3 days after the second MV infection. Ruxolitinib was administered orally (gavage), twice daily at $50 \mathrm{mg} / \mathrm{kg}$ (Figure $8 \mathrm{~A}$ ). Drug administration was well tolerated and mice continued to gradually gain weight (Figure 8B). To evaluate the impact of JAK/STAT signaling inhibition at the GBM tumor-immune microenvironment, some of the mice (4-5 per group) were euthanized for correlative analysis when 2 animals from the inactivated MV-s-NAP-uPA plus DMSO plus isotype control group displayed symptoms of GBM disease progression. The remaining mice were used for survival efficacy assessment (Figure 8A). A higher percentage (87.5\%) of mice that received MV-s-NAP-uPA plus anti-PD1 plus ruxolitinib triple therapy were long-term survivors compared with $62.5 \%$ in the group of mice that received MV-s-NAP-uPA plus anti-PD1. MV-s-NAP-uPA plus ruxolitinib combination therapy cured $25 \%$ of the CT-2A tumor-bearing mice, suggesting a synergistic action (Figure 8C and Table 3).

Notably, PD-L1 expression on myeloid cells and particularly on myeloid-derived suppressor cells (MDSCs) in the brain was almost completely abrogated with MV-s-NAP plus ruxolitinib plus anti-PD1 combination treatment (Figure 8D). Similarly, MVmediated myeloid cell recruitment and upregulation of the terminal differentiation marker KLRG1 on myeloid cells in the brain was reduced. Triple therapy impeded effector $\mathrm{T}$ cell function, as evidenced by reduced KLRG1 and PD1 expression on $\mathrm{CD}^{+} \mathrm{T}$ cells in the brain. Interestingly, the total number of splenic myeloid cells and MDSCs as well as PD-L1 expression on these cells were not affected by triple therapy (Supplemental Figure 10). Therefore, the PD1/PD-L1 pathway and the JAK1/JAK2 signaling pathway constitute complementary therapeutic targets for immunovirotherapy of GBM.

\section{Discussion}

Following the FDA's first approval of an OV for cancer treatment in 2015, oncolytic virotherapy has emerged as a viable clinical approach for cancer treatment (39). Our group has first introduced oncolytic MV vectors to clinical trials and have demonstrated that they are an attractive platform in cancer virotherapy with oncolytic activity and ability to elicit antitumor immunity. We hypothesized that MV-Edm vaccine strains armed with $H$. pylori NAP antigen could deliver an enhanced immunostimulatory cargo in 

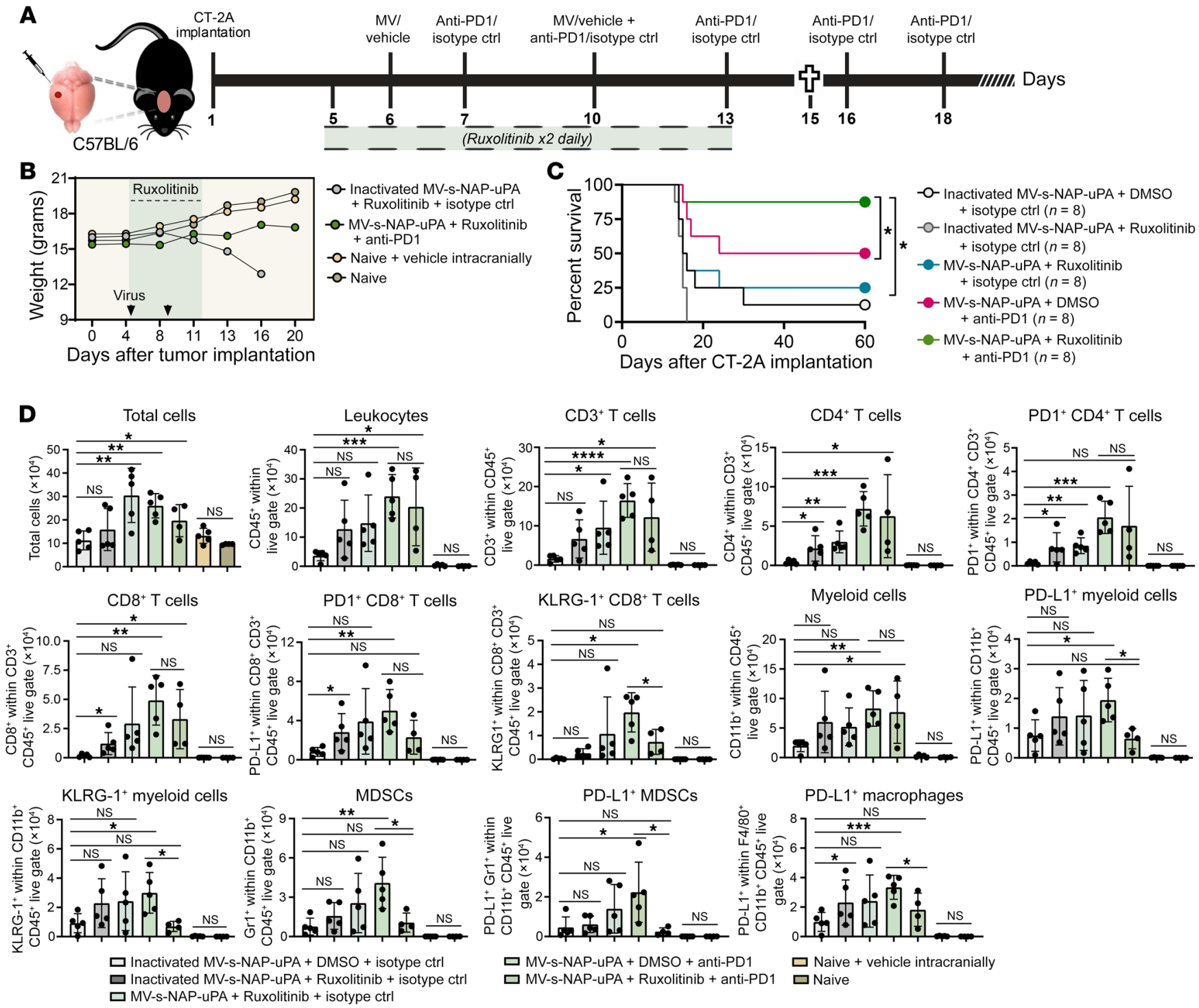

$\square$ MV-s-NAP-uPA + DMSO + anti-PD1 $\square$ Naive + vehicle intracranially $\square$ MV-s-NAP-uPA + Ruxolitinib + isotype ctr

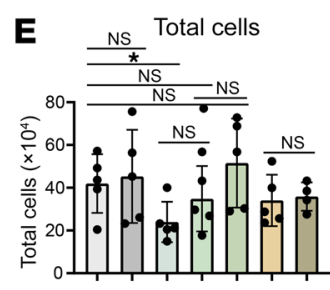

$\mathrm{CD}^{+} \mathrm{T}$ cells

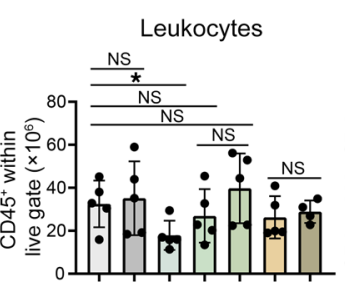

$\mathrm{PD}^{+}{ }^{+} \mathrm{CD} 8^{+} \mathrm{T}$ cells
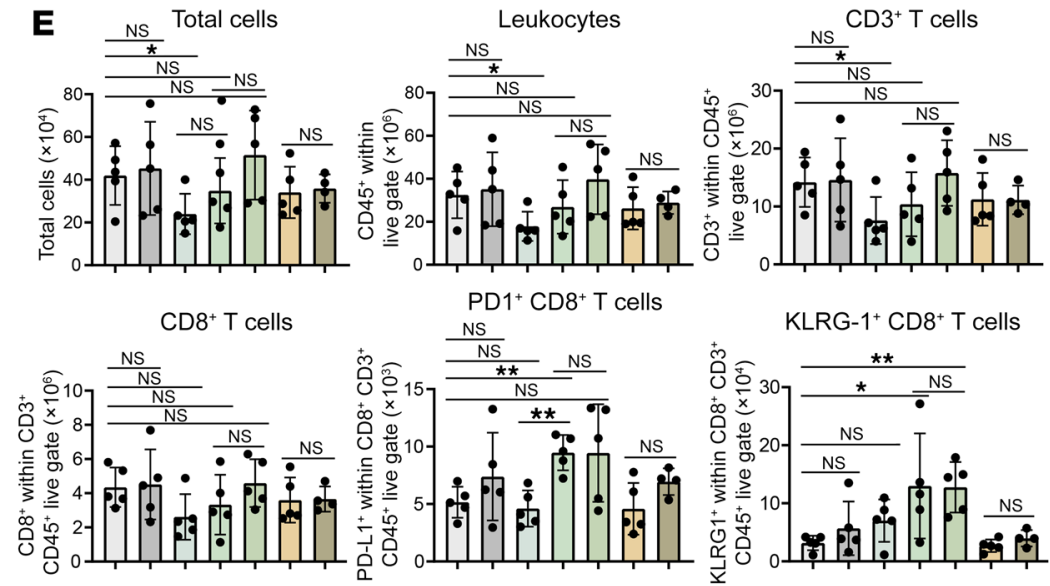

$\mathrm{KLRG}-1^{+} \mathrm{CD} 8^{+} \mathrm{T}$ cells
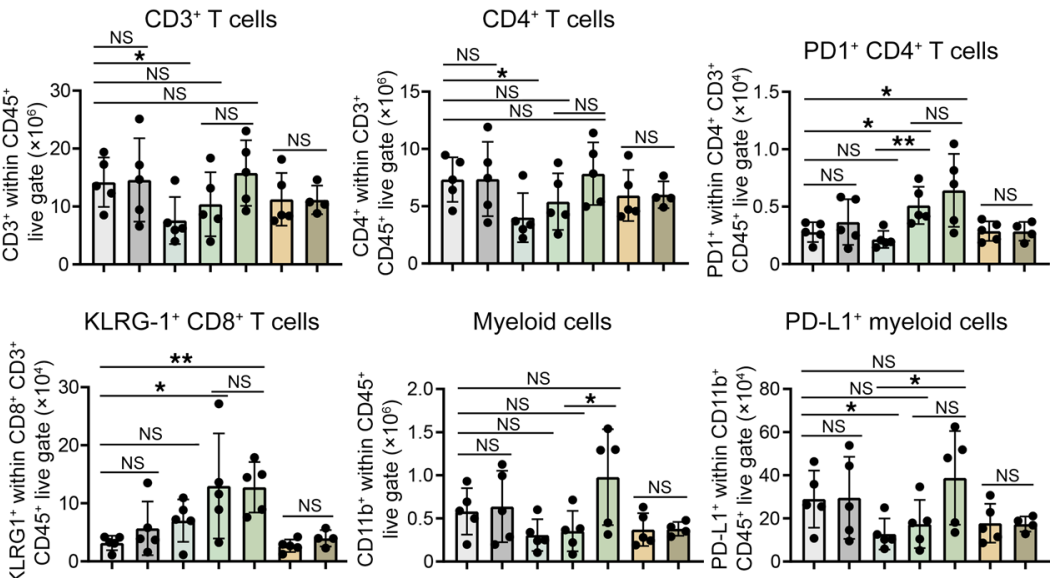

Myeloid cells

PD-L1+ myeloid cells

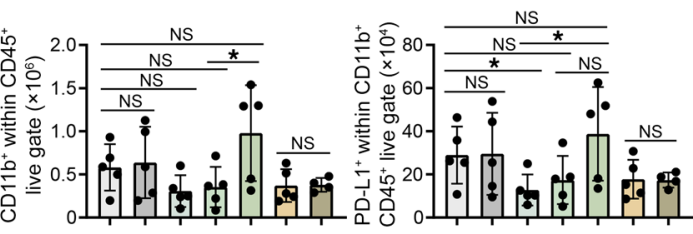

口 Inactivated MV-s-NAP-uPA + DMSO + isotype ctrl $\square$ Inactivated MV-s-NAP-uPA + Ruxolitinib + isotype ctr| 口 MV-s-NAP-uPA + Ruxolitinib + isotype ctrl 口 MV-s-NAP-uPA + DMSO + anti-PD1

$\square$ MV-s-NAP-uPA + Ruxolitinib + anti-PD1 $\square$ Naive + vehicle intracranially $\square$ Naive 
Figure 8. The JAK1/JAK2 signaling pathway inhibitor ruxolitinib improves cure rate of MV-s-NAP-uPA plus anti-PD1-treated mice. (A) C57BL/6 mice bearing CT-2A gliomas were treated as described in the schema. Mice received $50 \mathrm{mg} / \mathrm{kg}$ ruxolitinib or vehicle (DMSO) twice daily by oral gavage. Ruxolitinib administration started 1 day before viral therapy and was terminated 3 days after the last viral injection. (B) Body weights (grams) of animals receiving various combination treatments. (C) Survival outcome of mice bearing CT-2A glioma and treated as described. Kaplan-Meier survival curves and Benjamini and Hochberg adjustment for multiple comparisons were used to calculate median survival times and compare survival between animal groups ( $n=8$ mice per group). (D) Cellular immune response was quantified in the brain of mice harboring CT-2A tumors. Animals were sacrificed for correlative analysis when 2 mice from the inactivated MV-s-NAP-uPA plus DMSO plus isotype control group exhibited GBM disease symptoms. Mice were perfused and individual brains were collected and analyzed by flow cytometry ( $n=4-5$ mice per group). All values represent mean $\pm S D$. ${ }^{*} P<0.05 ;{ }^{* *} P<0.01 ;{ }^{* * *} P<$ $0.001 ;{ }^{* * *} P<0.0001$ by 2-way ANOVA followed by Tukey's multiple comparison test. NS, not significant. Detailed statistical differences between groups are presented in Table 3.

clinically relevant syngeneic murine models and human PDXs of orthotopic GBM and enhance sensitivity to immune checkpoint inhibitors. Despite limited replication of retargeted MV strains in murine GBM cells, we demonstrated that arming MV with NAP further enhanced the release of inflammatory mediators following viral infection and promoted systemic antitumor responses. These responses could be effectively augmented by systemic antiPD1 therapy, resulting in improvement of the tumor cure rate via a $\mathrm{CD}^{+} \mathrm{T}$ cell-mediated mechanism.

Despite the promise associated with clinical use of immunooncology approaches including immune checkpoint inhibitors, less than $40 \%$ of cancer patients are candidates for these approaches and less than $20 \%$ of patients derive clinical benefit $(40,41)$. Here, we show that bacterial antigens represent a powerful source of immunostimulatory agonists in the context of GBM that can boost immunogenicity of OVs and enhance the modest efficacy or lack of efficacy of immune checkpoint inhibitors. We demonstrated that MV-s-NAP infection of glioma cells results in immunogenic cell death with an increase in MHC class I expression and release of DAMPs (CALR, HSP90, and HMGB1) by infected cells. Upregulation of MHC class I by glioma cells has been linked to better tumor prognosis, and higher susceptibility to $\mathrm{CD} 8^{+} \mathrm{T}$ cell-mediated lysis (42). In parallel, induction of immunogenic cell death has been a keystone of antitumor immunity of certain OVs, often associated with immune-mediated tumor clearance (43-45). We showed that the antitumor effect in immunocompetent mice bearing orthotopic CT-2A and GL261 GBM led to immunogenic cell death accompanied by increased infiltration of $\mathrm{CD} 45^{+}$leukocytes, macrophages, and cytotoxic $\mathrm{CD} 8^{+} \mathrm{T}$ cells in the brain and a systemic immune activation. Combination immunovirotherapy was able to overcome limitations of single-agent therapy and provide potent synergistic responses. Using mice bearing CT-2A tumors in both hemispheres we also demonstrated an abscopal effect of the MV-s-NAP-uPA/anti-PD1 combination. Our findings suggest that the NAP bacterial TLR2 agonist expressed in OV platforms can manipulate the innate immune signaling and, following killing of tumor cells, stimulate proinflammatory conditions that alter the TME. This leads to boosting of the host antitumor immunity and creates synergy with immune checkpoint blockade regimens; these findings generate optimism for the therapeutic potential of this approach in GBM treatment.

The requirement of a $\mathrm{CD} 8^{+} \mathrm{T}$ cell-mediated response for enhanced survival in the MV-s-NAP-uPA plus anti-PD1 therapy group was supported by our results showing that depletion of $\mathrm{CD} 8^{+}$ $\mathrm{T}$ cells can lead to complete loss of the survival benefit, whereas neutrophils, $\mathrm{CD} 4^{+}$cells, and NK cells were unlikely to represent a major determinant of the observed antitumor response, thus highlighting the central role of $\mathrm{T}$ cell-mediated immunity in mediating the immunotherapeutic effect (46-48). The capacity of NAP to induce a monocyte-enriched milieu has possibly contributed to the Th1 antitumor activity $(49,50)$. In addition, potential activation of brain tissue-resident immune cells cannot be excluded. Whether crosstalk between innate immune cells in the brain such as microglia and macrophages with $\mathrm{CD} 8^{+} \mathrm{T}$ cells has contributed to the antitumor immune response elicited by localized MV-NAP therapy will require further investigation.

Inhibition of the JAK/STAT signaling pathway with ruxolitinib enhanced viral replication and induction of immunogenic cell death in all GBM models tested in vitro, representing a rational approach to overcome resistance to MV infection and create immunostimulatory signals in immune-silent brain tumors. Our experiments showed that secretion of DAMPs increased following oncolytic MV infection in the presence of ruxolitinib, suggesting that increased replication can augment the immunostimulatory effect. Prolonged exposure to IFN type I and II orchestrates resistance to immune checkpoint blockade therapy (51-53). For example, in clinical studies, JAK1 or JAK2 mutations were detected in progressive melanoma lesions following development of resistance to immune checkpoint inhibitor therapy $(54,55)$. PD-L1 expression in MDSCs is also regulated by autocrine type I IFN (51). Long-term blocking of IFN signaling on host cells, however, could interfere with the generation of antitumor $(56)$ and antiviral responses $(57,58)$ and decrease virus-induced PD-L1 expression (59). Markedly, blocking type I IFN activation with the JAK/STAT inhibitor ruxolitinib downregulated virus-induced PD-L1 expression on MDSCs in the brain, leading to enhanced survival benefit in the CT-2A glioma-bearing mice. In parallel, ruxolitinib impaired effector $\mathrm{T}$ cell function, as evidenced by reduced KLRG1 and PD1 expression on $\mathrm{CD} 8^{+} \mathrm{T}$ cells in the brain. In a clinical case report of Merkel cell carcinoma (MCC), immune checkpoint inhibitor therapy with nivolumab in combination with ruxolitinib phosphate achieved a sustained complete metabolic response (60). These findings indicate that a combination of antiPD1 therapy and ruxolitinib can have synergistic potential; nevertheless, timing is critical. Immunotherapeutic strategies that aim to include ruxolitinib or other JAK inhibitors should be carefully timed when combined with immune checkpoint blockade therapies that are regulated through IFN signaling, since prolonged duration of exposure can result in immunosuppression. Although not within the scope of this publication, we are in the process of evaluating optimal duration of ruxolitinib as part of this combinatorial strategy.

The findings presented in this study can have direct translational relevance since MV-Edm vaccine platforms are current- 


\section{Table 3. Statistical analysis of survival outcomes in the CT-2A glioblastoma model following treatment with MV \pm anti-PD1 \pm ruxolitinib combination therapy}

CT-2A model

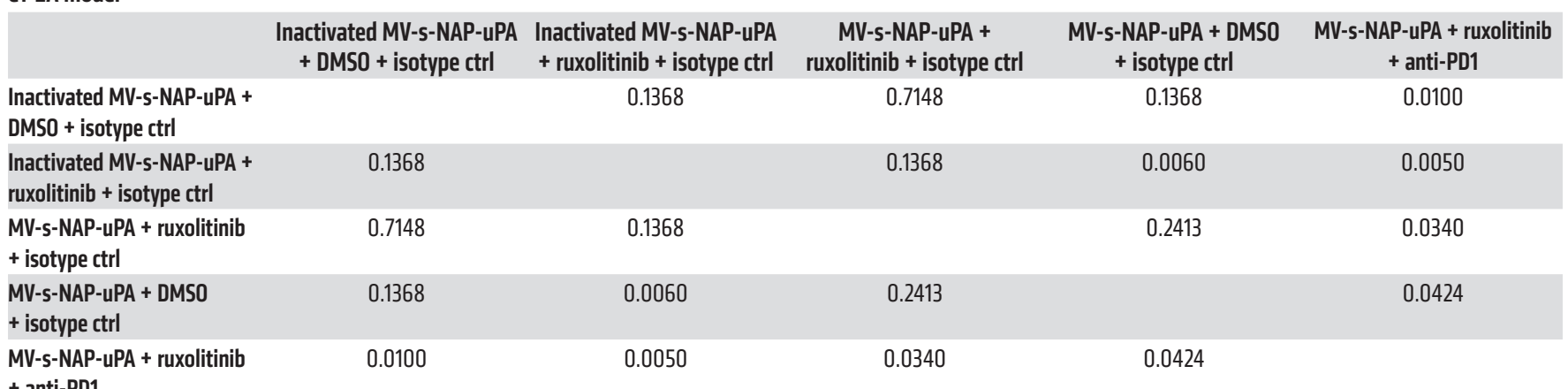

+ anti-PD1

The Benjamini and Hochberg false discovery rate (FDR) method was used to adjust for multiple comparisons. Statistical significance was set at FDR and $P$ values $<0.05$.

ly being evaluated in clinical trials for GBM (ClinicalTrials.gov: NCT00390299) with excellent safety and early evidence of biologic activity, while anti-PD1 antibodies such as nivolumab and pembrolizumab are FDA approved for the treatment of solid tumors (7). Pilot toxicology studies of MV-NAP in transgenic IFNAR-KO CD46-Ge mice (61) (which express the MV CD46 receptor in a distribution similar to that in humans) have shown lack of toxicity (unpublished observations from our group), further supporting the rationale of a phase I clinical trial in GBM patients. A possible challenge for using MV-Edm therapeutically in cancer patients is that most patients have preexisting $\mathrm{T}$ and $\mathrm{B}$ cell immunity to MV due to measles vaccination in childhood. To bypass preexisting immunity and viral exclusion by the blood-brain barrier, targeted tumor injection directly into the tumor or tumor cavity has been shown to be well tolerated. Injecting directly into the tumor cavity can prevent serum neutralization and systemic toxicity, and would potentially modulate the TME more efficiently as compared with systemic virus administration (62).

This study is the first to our knowledge to report that bacterial antigen-armed oncolytic MV strains lead to an antitumor effect, tumor immune recognition, and a durable antitumor response when combined with anti-PD1 immune checkpoint blockade therapy and ruxolitinib, which is far superior to monotherapy and can possibly be used to reverse primary resistance to immune checkpoint blockade in GBM. These findings provide strong support for clinical translational strategies of immunostimulatory MV-s-NAP in tandem with anti-PD1 immune checkpoint blockade and JAK1/ JAK2 inhibitors in GBM treatment.

\section{Methods}

Mice. Female C57BL/6 and BALB/c nude mice 4-5 weeks old were obtained from Taconic Biosciences and housed under specific pathogen-free conditions at the Mayo Animal Care Facility. Mice were 5-7 weeks old at the beginning of the experiments.

Production of UPAR-retargeted MV-s-NAP. Generation of recombinant MV fully retargeted to mouse UPAR with ablated CD46 and SLAM receptor binding sites of hemagglutinin-retargeted virus has been previously described (63). This construct included a second SpeI site that prevented insertion of the modified hemagglutinin protein using the PacI/SpeI digestion sites. Thus, we modified the MV-s-NAP vector by replacing a fragment from the MV-GFP strain with a single SpeI site using SalI digestion that allowed insertion of the recombinant hemagglutinin. The receptor-binding uPA fragment was digested with NotI/SfiI restriction enzymes and subcloned into MV-Edm strain hemagglutinin, as described previously (64). The retargeted hemagglutinin was subsequently subcloned in the MV-s-NAP fulllength plasmid between PacI and SpeI restriction enzyme sites and rescued as described using the 293-3-46 cell rescue system (65). Schematic representation of the UPAR-retargeted and control MV strains used in the experiments are shown in Figure 1A. MV-s-NAP-uPA and MV-GFP-uPA constructs were designed to encode a chimeric secretory NAP (s-NAP) transgene or a green fluorescent protein (GFP) reporter, respectively (21). Recombinant strains were propagated on Vero cells and viral stocks were stored at $-80^{\circ} \mathrm{C}$. Viral titer was determined in plaque-forming units and tissue culture infectious doses $50 \%\left(\right.$ TCID $\left._{50}\right)$ per $\mathrm{mL}$.

Cell lines. Vero (African green monkey kidney; ATCC), CT-2A (a gift from Richard Vile's lab, Mayo Clinic), and GL261 (a gift from Aaron Johnson's laboratory, Mayo Clinic) syngeneic murine GBM cells were grown adherent to cell culture flasks (Corning) in Dulbecco's modified Eagle's medium (DMEM) (Gibco, Life Technologies) containing $10 \%$ inactivated fetal bovine serum (FBS) (Corning) supplemented with $1 \%$ L-glutamine and $1 \%$ penicillin/streptomycin (Corning) in a humidified atmosphere containing $5 \% \mathrm{CO}_{2}$ at $37^{\circ} \mathrm{C}$. The J774A.1 mouse macrophage cell line (ATCC) on a BALB/c background and the B16-F10 melanoma cell line (a gift from the Vile lab) were cultured in DMEM supplemented as described above. Adherently cultured Vero, CT-2A, GL261, and B16-F10 cells were removed for passage after $0.25 \%$ trypsin (Corning) digestion and J774A.1 cells by scraping. Primary patient-derived GBM cell lines GBM6 and GBM12 (provided by Jann Sarkaria's lab, Mayo Clinic) were generated from resected human GBM patient tumors and maintained as subcutaneous xenografts and short-term in vitro cultures (66). GBM6 cells were maintained in DMEM containing $10 \%$ inactivated FBS and 
GBM12 cells in KnockOut DMEM/F-12 Basal Media supplemented with StemPro NSC SFM (Thermo Fisher Scientific), recombinant human basic FGF (Thermo Fisher Scientific), recombinant human EGF (Thermo Fisher Scientific), 1\% L-glutamine, and 1\% penicillin/ streptomycin and cultured on laminin-coated plates (Sigma-Aldrich). All media were filtered through a sterile filter/bottle (Corning) before use in experiments.

Virus cytotoxicity in vitro. The cytopathic potential of the uPAR-retargeted MV strains against GBM cells was determined by CellTiter-Blue Cell Viability Assay (Promega) and crystal violet staining. A detailed description of the procedure is presented in the supplemental material.

ELISA for detection of NAP transgene expression by infected cells. Secretory NAP release by MV-s-NAP-infected GBM cells was quantified in vitro by NAP-specific ELISA. More information can be found in the online supplemental material.

Cytokine detection by multiplex assay. Cytokine release was examined in fresh, cell-free culture supernatant samples of CT-2A and GL261 glioma cells following 48 hours of in vitro infection with uPAR-retargeted MV-s-NAP, heat-inactivated MV-s-NAP, or MV-GFP at an MOI of 2. The BioLegend mouse LEGENDplex beadbased immunoassay (anti-virus response panel; catalog 740621) was used to quantify 13 soluble analytes (CXCL1, TNF- $\alpha$, MCP-1, IL-12p70, RANTES, IL-1 $\beta$, CXCL10, GM-CSF, IL-10, IFN- $\beta$, IFN- $\alpha$, IFN- $\gamma$, and IL-6). Results were acquired with the LSRFortessa Cytometer (BD Biosciences) and analyzed with LEGENDplex Data Analysis Software (BioLegend).

Assessment of immunogenic cell death. The ability of MV infection to induce immunogenic cell death was determined in vitro by measuring the release of 3 different DAMPs. Briefly $2 \times 10^{5}$ CT-2A, GL261, GBM6, and GBM12 cells were plated in triplicate in 6-well tissue culture-treated plates and subsequently infected at MOI 5 with recombinant MV strains in Opti-MEM $1 \times$ (Gibco) containing 2\% FBS and 1\% penicillin/ streptomycin. Following 4 hours of coincubation, media were replaced with complete culture media and 24, 48, and 72 hours later extracellular release of HMGB1, CALR, and HSP90 was assessed. Where indicated in the figure legends, cells were cotreated with $5 \mu \mathrm{M}$ ruxolitinib. To test HMGB1 release, cell-free supernatant was collected from triplicate samples following quick centrifugation at $2500 \mathrm{~g}$ for 5 minutes and stored at $-20^{\circ} \mathrm{C}$. HMGB1 release was determined following the HMGB1 ELISA kit (IBL International GMBH) instructions. In parallel, cells were harvested by scraping and processed for anti-CALR-PE (Abcam, catalog ab83220;1:400), anti-HSP90-PE (Novus Bio, catalog NB100-1972; 1:150), and IgG1-PE (BioLegend, catalog 400114; 1:150) surface labeling and quantification by flow cytometry. In a parallel experiment, cell culture supernatants cleared of debris were collected 24 and 48 hours after infection with MV strains at MOI 2 and MOI 5 for IFN- $\alpha$ and IFN- $\beta$ detection using the mouse IFN- $\alpha$ all subtypes ELISA Kit (LSBio) and the Legend Max mouse IFN- $\beta$ ELISA kit (BioLegend), respectively. Human IFN- $\beta$ was measured in tissue culture supernatant using a human IFN- $\beta$ ELISA kit (R\&D Systems). Where indicated, cells were treated for 24 hours with $500 \mathrm{U} / \mathrm{mL}$ exogenous recombinant mouse IFN- $\beta$ (PBL Assay Science, catalog 12400-1), murine IFN- $\gamma$ (PeproTech, catalog 315-05), or human IFN- $\gamma$ (PeproTech, catalog 30-02). For in vitro inhibition of IFNAR-1 expression, glioma cells were treated with $10 \mu \mathrm{g} / \mathrm{mL}$ mouse anti-IFNAR-1 blocking antibody (clone MAR1-5A3) obtained from BioXCell.
qPCR. uPAR-retargeted-MV titers were determined by probebased qPCR. The procedure is described in detail in the online supplemental material.

In vivo treatments. Orthotopic tumors were established by implantation of $2 \times 10^{5} \mathrm{CT}-2 \mathrm{~A}, 3 \times 10^{5}$ GL261, and $3 \times 10^{4}$ B16-F10 cells in immunocompetent C57BL/6 female mice and $3 \times 10^{6}$ GBM6 or GBM12 cells in BALB/c nude female mice in $5 \mu \mathrm{L}$ sterile PBS, into the caudate nucleus, using a small animal stereotactic frame (SI Instruments) and a 26-gauge Hamilton syringe (Hamilton Company). On day 5 after tumor implantation, mice were randomized and treated intracranially/intratumorally with $2 \times 10^{5} \mathrm{TCID}_{50}$ MV-s-NAP, heatinactivated MV-s-NAP, MV-s-NAP-uPA, heat-inactivated MV-s-NAPuPA, or MV-GFP-uPA in a total volume of $10 \mu \mathrm{L}$ Opti-MEM using the same coordinates as for cell implantation. Heat inactivation was performed at $90^{\circ} \mathrm{C}$ for 30 minutes in a PCR machine. Surgical procedures and intratumoral administration of saline (PBS) was also performed in parallel in healthy, naive, non-tumor-bearing mice to serve as controls. Murine anti-PD1 (CD279; clone RPMI-14, catalog 114115) or isotype control (rat IgG2a, א; clone RTK2758, catalog 400564) both obtained from BioLegend were administered intraperitoneally (i.p.) at $200 \mu \mathrm{g} / \mathrm{mouse}$ in sterile PBS. Ruxolitinib treatment was initiated a day before the start of virus treatment and was provided twice daily at 50 $\mathrm{mg} / \mathrm{kg}$ dose by oral gavage and was terminated 48-72 hours after the last MV administration. Tumor-bearing animals were monitored daily and sacrificed when they reached GBM humane endpoint criteria.

Antibody depletion of immune cell subsets. Anti-CD4 (clone GK1.5, catalog BE0003-1), anti-CD8a (clone 2.43, catalog BE0061), antiNK1.1 (clone PK136, catalog BE0036), anti-Ly6G (clone 1A, cata$\log$ BE0075), and anti-IgG [hamster IgG $\mathrm{F}\left(\mathrm{ab}^{\prime}\right)_{2}$ fragments; catalog BE0091-FAB] in vivo depleting mAbs were purchased from BioXCell. Anti-CD4, anti-CD8, and anti-NK1.1 mAbs were injected i.p. $(250 \mu \mathrm{g} /$ mouse) starting 1 day before initiation of the MV treatment and every 3 days thereafter. To eliminate neutrophils, mice were injected i.p. with $500 \mu \mathrm{g} /$ mouse anti-Ly6G following the same depletion schedule as described for the rest of the immune cell subsets. Immune cell depletion was terminated 30 days after the last anti-PD1 treatment. Successful depletion ( $>95 \%$ ) of the various immune cell subsets was verified by flow cytometric analysis of peripheral blood of recipient mice.

Flow cytometry staining. Established antibody panels and protocols were used for flow cytometric analysis of various immune cell populations. More information can be found in the online supplemental methods. FlowJo software (TreeStar) was used for data analysis. Immunophenotyping gating strategies of immune cells in the mouse brain, spleen, and peripheral blood are provided in Supplemental Figure 11.

Statistics. All statistical comparisons were performed with Prism software version 6.0 (GraphPad Software). Significance of differences between 2 groups was evaluated by unpaired, 2-tailed $t$ test. Multiple comparisons were analyzed using 1-or 2-way analysis of variance (ANOVA) with Tukey's and Dunnett's post hoc multiple comparison tests (67). Kaplan-Meyer survival curves were used to assess animal survival, and comparisons were performed using the log-rank Mantel-Cox test (68) and Benjamini and Hochberg false discovery rate (FDR) (69) for correction for multiple comparisons. Statistical significance was set at FDR and $P$ values less than 0.05 and not significant (NS) greater than 0.05 .

Graphical abstract. The graphical abstract was made using BioRender (https://biorender.com/). 
Study approval. All mouse experiments and surgical procedures were approved by the Mayo Institutional Animal Care and Use Committee (IACUC) and met the requirement of the Mayo Animal Care Facility and the NIH Guide for the Care and Use of Laboratory Animals (National Academies Press, 2011). Primary patient-derived GBM cell lines, GBM6 and GBM12, were generated from resected human GBM patient tumors under a Mayo Clinic IRB-approved protocol (Mayo Clinic IRB 06-004440).

\section{Author contributions}

EP designed and performed experiments, analyzed data, and prepared the manuscript. CK, KV, AA, and IDI performed the experiments and assisted with data analysis and interpretation. SS, KC, KA, SKA, and AJJ assisted with data analysis and interpretation. EG supervised experimental design, data analysis, and assisted with manuscript preparation. All authors reviewed and approved the final manuscript.

\section{Acknowledgments}

We would like to thank Jaime Merchan (University of Miami) for providing the MV plasmid backbone encoding hemagglutinin retargeted to mouse UPAR and the laboratories of Jann Sarkaria and Richard Vile for their generous gifts of GBM cell lines used in this study. Finally, we would to thank the Flow Cytometry, Pathology, and Microscopy research core facilities at the Mayo Clinic for technical assistance. This study was supported by NIH grants (R01 CA258239 and R01 CA154348 to EG), Mayo Clinic Brain and Breast Spore grants from the NIH (P50 CA108961 and P50 116201 to EG), and The Ben and Catherine Ivy Foundation.

Address correspondence to: Evanthia Galanis, Department of Molecular Medicine, Mayo Clinic, 200 First Street SW, Rochester, Minnesota 55905, USA. Phone: 507.202.7652; Email: galanis. evanthia@mayo.edu.
1. Ostrom QT, et al. CBTRUS statistical report: primary brain and other central nervous system tumors diagnosed in the United States in 20122016. Neuro Oncol. 2019;21(suppl 5):v1-v100.

2. Aldape K, et al. Challenges to curing primary brain tumours. Nat Rev Clin Oncol. 2019;16(8):509-520.

3. Friebel E, et al. Single-cell mapping of human brain cancer reveals tumor-specific instruction of tissue-invading leukocytes. Cell. 2020;181(7):1626-1642.

4. Russell SJ, Barber GN. Oncolytic viruses as antigen-agnostic cancer vaccines. Cancer Cell. 2018;33(4):599-605.

5. Twumasi-Boateng $\mathrm{K}$, et al. Oncolytic viruses as engineering platforms for combination immunotherapy. Nat Rev Cancer. 2018;18(7):419-432.

6. Msaouel P, et al. Clinical trials with oncolytic measles virus: current status and future prospects. Curr Cancer Drug Targets. 2018;18(2):177-187.

7. Galanis E, et al. Phase I/II trial of adipose tissue derived mesenchymal stem cell delivery of a measles virus strain engineered to express the sodium iodide symporter in ovarian cancer patients. Mol Ther. 2020;28:4s1:589 [abstract 1370]. https://doi.org/10.1016/j. ymthe.2020.04.019.

8. Kurokawa C, et al. Constitutive interferon pathway activation in tumors as an efficacy determinant following oncolytic virotherapy. J Natl Cancer Inst. 2018;110(10):1123-1132.

9. Kurokawa C, Galanis E. Interferon signaling predicts response to oncolytic virotherapy. Oncotarget. 2019;10(16):1544-1545.

10. Msaouel P, et al. Oncolytic measles virus retargeting by ligand display. Methods Mol Biol. 2012;797:141-162.

11. Smith HW, Marshall CJ. Regulation of cell signalling by uPAR. Nat Rev Mol Cell Biol. 2010;11(1):23-36.

12. Jing $\mathrm{Y}$, et al. In vivo safety, biodistribution and antitumor effects of uPAR retargeted oncolytic measles virus in syngeneic cancer models. Gene Ther. 2014;21(3):289-297.

13. Gilder AS, et al. The urokinase receptor induces a mesenchymal gene expression signature in glioblastoma cells and promotes tumor cell survival in neurospheres. Sci Rep. 2018;8(1):2982.

14. Hu J, et al. Crosstalk between the urokinase-type plasminogen activator receptor and EGF receptor variant III supports survival and growth of glioblastoma cells. Proc Natl Acad Sci U S A. 2011;108(38):15984-15989.

15. Forbes NS. Engineering the perfect (bacterial) cancer therapy. Nat Rev Cancer. 2010;10(11):785-794

16. Cronin M, et al. Bacterial-mediated knockdown of tumor resistance to an oncolytic virus enhances therapy. Mol Ther. 2014;22(6):1188-1197.

17. Satin B, et al. The neutrophil-activating protein (HP-NAP) of Helicobacter pylori is a protective antigen and a major virulence factor. J Exp Med. 2000;191(9):1467-1476.

18. Amedei A, et al. The neutrophil-activating protein of Helicobacter pylori promotes Th1 immune responses. J Clin Invest. 2006;116(4):1092-1101.

19. Iankov ID, et al. Measles virus expressed Helicobacter pylori neutrophil-activating protein significantly enhances the immunogenicity of poor immunogens. Vaccine. 2013;31(42):4795-4801.

20. Iankov ID, et al. Immunogenicity of attenuated measles virus engineered to express Helicobacter pylori neutrophil-activating protein. Vaccine. 2011;29(8):1710-1720.

21. Iankov ID, et al. Expression of immunomodulatory neutrophil-activating protein of Helicobacter pylori enhances the antitumor activity of oncolytic measles virus. Mol Ther. 2012;20(6):1139-1147.

22. Schwartz DM, et al. JAK inhibition as a therapeutic strategy for immune and inflammatory diseases. Nat Rev Drug Discov. 2017;16(12):843-862.

23. Kijima T, et al. STAT3 activation abrogates growth factor dependence and contributes to head and neck squamous cell carcinoma tumor growth in vivo. Cell Growth Differ. 2002;13(8):355-362.

24. Verstovsek $S$, et al. Long-term treatment with ruxolitinib for patients with myelofibrosis: 5 -year update from the randomized, double-blind, placebo-controlled, phase 3 COMFORT-I trial. J Hematol Oncol. 2017;10(1):55.

25. Kiladjian JJ, et al. Long-term efficacy and safety of ruxolitinib versus best available therapy in polycythaemia vera (RESPONSE): 5 -year follow up of a phase 3 study. Lancet Haematol. 2020;7(3):e226-e237.

26. Heine A, et al. The JAK-inhibitor ruxolitinib impairs dendritic cell function in vitro and in vivo. Blood. 2013;122(7):1192-1202.

27. Luo W, et al. Targeting JAK-STAT signaling to control cytokine release syndrome in COVID-19. Trends Pharmacol Sci. 2020;41(8):531-543.

28. Hurwitz HI, et al. Randomized, double-blind, phase ii study of ruxolitinib or placebo in combination with capecitabine in patients with metastatic pancreatic cancer for whom therapy with gemcitabine has failed. JClin Oncol. 2015;33(34):4039-4047.

29. McLean K, et al. Leukemia inhibitory factor functions in parallel with interleukin- 6 to promote ovarian cancer growth. Oncogene. 2019;38(9):1576-1584.

30. Patel MR, et al. JAK/STAT inhibition with ruxolitinib enhances oncolytic virotherapy in nonsmall cell lung cancer models. Cancer Gene Ther. 2019;26(11-12):411-418.

31. Wang W, et al. Transcriptional regulation of antiviral interferon-stimulated genes. Trends Microbiol. 2017;25(7):573-584.

32. Galluzzi L, et al. Consensus guidelines for the definition, detection and interpretation of immunogenic cell death. J Immunother Cancer. 2020;8(1):e000337.

33. Liu YC, et al. TLR2 signaling depletes IRAK1 and inhibits induction of type I IFN by TLR7/9. Jimmunol. 2012;188(3):1019-1026.

34. Garrido F, et al. The urgent need to recover MHC class I in cancers for effective immunotherapy. Curr Opin Immunol. 2016;39:44-51.

35. Villarino AV, et al. Mechanisms and consequences of Jak-STAT signaling in the immune system. Nat Immunol. 2017;18(4):374-384.

36. Davidson TB, et al. Expression of PD-1 by T cells in malignant glioma patients reflects exhaustion and activation. Clin Cancer Res. 2019;25(6):1913-1922.

37. Charlton JJ, et al. Programmed death-1 shapes memory phenotype CD8 T cell subsets in a cell-intrinsic manner. J Immunol. 2013;190(12):6104-6114.

38. Chongsathidkiet $P$, et al. Sequestration of $T$ cells in bone marrow in the setting of glioblastoma and other intracranial tumors. Nat Med. 
2018;24(9):1459-1468.

39. Andtbacka RH, et al. Talimogene laherparepvec improves durable response rate in patients with advanced melanoma. JClin Oncol. 2015;33(25):2780-2788.

40. Weller M, et al. Rindopepimut with temozolomide for patients with newly diagnosed, EGFRvIII-expressing glioblastoma (ACT IV): a randomised, double-blind, international phase 3 trial. Lancet Oncol. 2017;18(10):1373-1385.

41. Reardon DA, et al. Effect of nivolumab vs bevacizumab in patients with recurrent glioblastoma: the checkmate 143 phase 3 randomized clinical trial. JAMA Oncol. 2020;6(7):1003-1010.

42. Yang W, et al. MHC class I dysfunction of glioma stem cells escapes from CTL-mediated immune response via activation of $\mathrm{Wnt} / \beta$-catenin signaling pathway. Oncogene. 2020;39(5):1098-1111.

43. Hardcastle J, et al. Immunovirotherapy with measles virus strains in combination with antiPD-1 antibody blockade enhances antitumor activity in glioblastoma treatment. Neuro Oncol. 2017;19(4):493-502.

44. Ma J, et al. Characterization of virus-mediated immunogenic cancer cell death and the consequences for oncolytic virus-based immunotherapy of cancer. Cell Death Dis. 2020;11(1):48.

45. Jiang $\mathrm{H}$, et al. Oncolytic adenovirus and tumor-targeting immune modulatory therapy improve autologous cancer vaccination. Cancer Res. 2017;77(14):3894-3907.

46. Lang FF, et al. Phase I study of DNX-2401 (Delta24-RGD) oncolytic adenovirus: replication and immunotherapeutic effects in recurrent malignant glioma. JClin Oncol. 2018;36(14):1419-1427.

47. Martínez-Vélez N, et al. The oncolytic virus Delta24-RGD elicits an antitumor effect in pediatric glioma and DIPG mouse models. Nat Commun. 2019;10(1):2235.

48. Zamarin D, et al. PD-L1 in tumor microenvironment mediates resistance to oncolytic immunotherapy. JClin Invest. 2018;128(4):1413-1428.

49. Oppmann B, et al. Novel p19 protein engages IL-12p40 to form a cytokine, IL-23, with biological activities similar as well as distinct from IL-12 Immunity. 2000;13(5):715-725.

50. D'Elios MM, et al. The neutrophil-activating protein of Helicobacter pylori (HP-NAP) as an immune modulating agent. FEMS Immunol Med Microbiol. 2007;50(2):157-164.

51. Xiao W, et al. IFNAR1 Controls autocrine type I IFN regulation of PD-L1 expression in myeloid-derived suppressor cells. JImmunol. 2018;201(1):264-277.

52. Benci JL, et al. Tumor interferon signaling regulates a multigenic resistance program to immune checkpoint blockade. Cell.2016;167(6):1540-1554.

53. Prestipino A, et al. Oncogenic JAK2(V617F) causes PD-L1 expression, mediating immune escape in myeloproliferative neoplasms. Sci Transl Med. 2018;10(429):eaam7729.

54. Zaretsky JM, et al. Mutations associated with acquired resistance to $\mathrm{PD}-1$ blockade in melanoma. N Engl JMed. 2016;375(9):819-829.

55 . Shin DS, et al. Primary resistance to PD-1 blockade mediated by JAK1/2 mutations. Cancer Discov. 2017;7(2):188-201.

56. Diamond MS, et al. Type I interferon is selectively required by dendritic cells for immune rejection of tumors. J Exp Med. 2011;208(10):1989-2003.

57. Ng CT, et al. Blockade of interferon Beta, but not interferon alpha, signaling controls persistent viral infection. Cell Host Microbe. 2015;17(5):653-661.

58. Sandler NG, et al. Type I interferon respons- es in rhesus macaques prevent SIV infection and slow disease progression. Nature. 2014;511(7511):601-605.

59. Chen J, et al. Type I IFN protects cancer cells from $\mathrm{CD}^{+} \mathrm{T}$ cell-mediated cytotoxicity after radiation. J Clin Invest. 2019;129(10):4224-4238.

60. Debureaux PE, et al. Nivolumab combined with ruxolitinib: antagonism or synergy? Ann Oncol. 2018;29(5):1334-1335.

61. Mrkic B, et al. Measles virus spread and pathogenesis in genetically modified mice. J Virol. 1998;72(9):7420-7427.

62. Maroun J, et al. Designing and building oncolytic viruses. Future Virol. 2017;12(4):193-213.

63. Jing $\mathrm{Y}$, et al. Tumor and vascular targeting of a novel oncolytic measles virus retargeted against the urokinase receptor. Cancer Res. 2009;69(4):1459-1468.

64. Allen C, et al. Interleukin-13 displaying retargeted oncolytic measles virus strains have significant activity against gliomas with improved specificity. Mol Ther. 2008;16(9):1556-1564.

65. Radecke F, et al. Rescue of measles viruses from cloned DNA. EMBO J. 1995;14(23):5773-5784.

66. Sarkaria JN, et al. Use of an orthotopic xenograft model for assessing the effect of epidermal growth factor receptor amplification on glioblastoma radiation response. Clin Cancer Res. 2006;12(7 pt 1):2264-2271.

67. Maxwell SE, Delaney HD. Designing Experiments and Analyzing Data. Lawrence Erlbaum; 2004.

68. Mantel N. Evaluation of survival data and two new rank order statistics arising in its consideration. Cancer Chemother Rep. 1966;50(3):163-170.

69. Benjamini Y, Hochberg Y. Controlling the false discovery rate: a practical and powerful approach to multiple testing. J R Stat Soc B. 1995;57:289-300. 